\title{
The Nephroprotective Properties of Extracellular Vesicles in Experimental Models of Chronic Kidney Disease: a Systematic Review
}

\author{
Natalia Nowak ${ }^{1}$ (D) Masayuki Yamanouchi ${ }^{2} \cdot$ Eiichiro Satake $^{3}$
}

Accepted: 17 May 2021 / Published online: 10 June 2021

(c) The Author(s) 2021

\begin{abstract}
Extracellular vesicle (EV)-based therapy was hypothesized as a promising regenerative approach which has led to intensive research of EVs in various pathologies. In this study, we performed a comprehensive systematic review of the current experimental evidence regarding the protective properties of EVs in chronic kidney disease (CKD). We evaluated the EVbased experiments, EV characteristics, and effector molecules with their involvement in CKD pathways. Including all animal records with available creatinine or urea data, we performed a stratified univariable meta-analysis to assess the determinants of EV-based therapy effectiveness. We identified 35 interventional studies that assessed nephroprotective role of EVs and catalogued them according to their involvement in CKD mechanism. Systematic assessment of these studies suggested that EVs had consistently improved glomerulosclerosis, interstitial fibrosis, and cell damage, among different CKD models. Moreover, EV-based therapy reduced the progression of renal decline in CKD. The stratified analyses showed that the disease model, administered dose, and time of therapeutic intervention were potential predictors of therapeutic efficacy. Together, EV therapy is a promising approach for CKD progression in experimental studies. Further standardisation of EV-methods, continuous improvement of the study quality, and better understanding of the determinants of EV effectiveness will facilitate preclinical research, and may help development of clinical trials in people with CKD.
\end{abstract}

Keywords Extracellular vesicle $\cdot$ Exosome $\cdot$ Mesenchymal stem cell $\cdot$ MiRNA $\cdot$ Kidney $\cdot$ Chronic kidney disease . Protection · Systematic review

This article belongs to the Topical Collection: Special Issue on Exosomes and Microvesicles: from Stem Cell Biology to Translation in Human Diseases

Guest Editor: Giovanni Camussi

Natalia Nowak

nzuznowak@gmail.com; natalia.nowak@umb.edu.pl

1 Faculty of Medicine, Centre for Bioinformatics and Data Analysis, Medical University of Bialystok, Bialystok, Poland

2 Department of Nephrology and Laboratory Medicine Faculty of Medicine Institute of Medical, Pharmaceutical and Health Sciences Graduate School of Medical Sciences, Kanazawa University, Toranomon Hospital, Nephrology Center, Tokyo, Japan

3 Section on Genetics and Epidemiology, Research Division, Joslin Diabetes Center, Department of Medicine, Harvard Medical School, MA, Boston, USA

\section{Introduction}

Chronic kidney disease (CKD) is a serious public health concern that can lead to end-stage kidney disease (ESKD). It affects more than 0.5 billion people with a global prevalence approaching $10 \%[1,2]$. Despite advances in the understanding of the pathophysiology of the disease, its pharmacological treatment remains limited to supportive measures and strategies limiting CKD progression, with no therapeutic interventions specifically altering CKD cause.

CKD develops when kidney oxygen delivery is reduced. Kidney hypoxia amplifies, which is followed by an impaired regenerative capacity, induced inflammatory response, oxidative stress, cell damage, and progressive fibrosis of the kidney compartments. To counteract these processes, various pharmacological treatments have been developed. None of these, however, have been yet clinically proven to effectively alter CKD outcome [3-5]. Unfortunately, classical pharmacological approaches often ignore complexities 
and interconnections of overlapping disease-related mechanisms, narrowly influencing a single pathway involved in the disease pathogenesis. This limits their effectiveness and raises the risk of possible negative drug effects [2,3]. An alternative approach, that may help overcome the bottleneck between regenerative medicine and current pharmacological treatments, relies on a targeted delivery and modulation of the disease pathways through extracellular vesicle (EV)mediated transfer.

EVs are membranous structures released by various cell types. They encapsulate functional molecules (nucleic acids, proteins, metabolites) of the parental cells and deliver them throughout the body [6]. Because of this feature, EVs participate in intracellular communication, affecting injury and regeneration processes of the receptor cells [7, 8]. Experimental studies support the hypothesis that exogenously administered stem cell-derived EVs, are incorporated into the kidney cells and may participate in renal repair. On the other side, those EVs are free of the tumorigenic and immunogenic shortcomings of the cellular therapies; and as such, they are considered as safer and more feasible path towards future regenerative medicine $[9,10]$.

Recent publications have reported an evidence of significant EV therapeutic effect with regard to chronic, progressive kidney disease; and showed this effect is mediated via multiple mechanisms. However, no systematic synthesis of these data is yet available. Moreover, some of those publications pointed out discrepancies in this effect observed among study settings [10-13]. Differences in factors related to study design may have contributed to this discrepancy: (1) small study groups (2) different animal species (3) increased risk for systemic error owing to the problems in experimental design, and (4) confounding factors influencing the sensitivity of kidney regenerative processes to EV-specific stimuli. Inconsistencies in EVs in experimental CKD models may have also be caused by several factors of a biological nature, f. ex. differences in examined disease stage or by the interactions between multiple pathophysiological and protective processes, but also by different EV-protocols utilised in the studies [14] or by heterogeneous study models (i.e., different doses of EVs, xenogeneic vs allogenic EV transplant, evaluated time-points) [12,13], and differences in the local effect of vesicular molecules [15], and thus these inconsistencies require a systematic analysis of the results.

In this report, we investigated the mechanisms by which EVs (and the molecules contained within) accelerate recovery from kidney injury in experimental CKD models. We evaluated the study design, EV-specific experiments, and analysed the active molecules contained within EVs, and their involvement in CKD pathways. Until this end, we assessed the combined protective effect in 35 studies and reported our findings following PRISMA standards
[16]. Additionally, in this study we evaluated, for the first time, a combined therapeutic effect of the EV-based treatment on renal function decline estimates, and explored the potential influencing factors by stratification.

\section{Methods}

\section{Search Strategy and Study Inclusion}

PubMed, Cochrane, and Web of Science were systematically searched to identify all publications that have assessed EVs (small or larger EVs) in: (a) original research study in vitro and in vivo (rodent or larger animals) pre-clinical models with (b) interventional study design (c) in experimental settings of progressive kidney disease (d) where the assumed protective effects of EVs or EV-derived molecular components were the main focus of the study. The last searches were performed at the end of July, 2020. We have included only the studies which have investigated the nephroprotective properties of EVs. We have downloaded a list of studies with the following query: (((extracellular vesicle* OR EV OR exosome* OR microvesicle) AND (kidney* OR renal* OR nephro* OR CKD* OR DKD* OR UUO) AND (protection* OR repair OR prevent OR ameliorate))). The references of articles were also screened for potentially relevant studies. Additional searches were performed manually and on the Exocarta database. During screening, the articles not meeting the above criteria were excluded. Articles were selected through reading title and abstract, and if these were not informative enough, the full article was screened for eligibility. Articles were discussed between all authors before exclusion. The exclusion criteria for the studies were the following: (a) Not an interventional study (e.g. the extracellular vesicles were not directly administered as a therapy; (b) No research performed (e.g. book chapter, review article, editorial, comment, etc.); (c) Animal model of acute kidney injury (e.g. ischemia-reperfusion injury and toxic AKI models (e.g. cisplatin, glycerol, gentamycin, folic acid, lipopolysaccharide) or AKI-CKD transition), kidney stone formation; (d) No relevant data. For selected studies, the full-text articles were then extracted and further analysed. No date or location restrictions were applied. To perform the quantitative meta-analyses, we needed to exclude a) studies [40, 41, 44], which examined genetically modified EVs as miRNA delivery vectors, $b$ ) in vitro study that evaluated EV miRNA [31], c) studies that did not evaluated outcomes of GFR decline [17, 19, 35, 37], and d) a study that did not reported size of the experimental groups [44]. The remaining studies were used for extracting GFR, blood creatinine, and blood urea. 


\section{Methods of Data Extraction}

Qualitative and quantitative data were extracted for metaanalyses and/or narrative synthesis. This included information on the therapeutic intervention (including source and size of administered vesicles; route, dosing and time-point of treatment administration; vesicle content), disease model (including CKD model, animal species, strain, gender, age), and outcome results. The outcome results for meta-analyses were serum creatinine, GFR, and serum urea. All studies with outcome data and the number of experimental groups were used for extracting sample size, and mean with standard deviation (SD) of each estimate to generate standardised mean differences (SMD). For studies which reported standard error of the mean (SEM), these were converted to standard deviations ( $\mathrm{SD}$, where $\mathrm{SD}=\sqrt{ } \mathrm{n} \times \mathrm{SEM})$. In a case of serial measurements, the last timepoint of the measurement for the same animals was evaluated (unless it was stated that animals were lost towards the end of the study). For studies that did not show the corresponding results in the main text, the figure calibration macro (Hessman) within ImageJ software (https://imagej.nih.gov/ij/plugins/\#tools) was utilised to extract data from the graphics. The outcome results for the systematic review were recorded under the following main categories: fibrosis, inflammation, cell damage, and oxidative stress.

\section{Quality Assessment}

To assess the methodological quality of the included experimental studies, we adapted the main criteria suggested by ISEV (MISEV, 2018). In line with these criteria, we assessed the quality of EV-included experiments by screening the concentration methods, the methodology to assess EV morphology (presence of EV enriched markers, size distribution, publication of TEM images to visualise the preparations and confirm the presence of EV population). In a formal bias analysis, we assessed study quality using the selected items from the Collaborative Approach to Metaanalysis and Review of Animal Data in Experimental Studies (CAMRADES) risk of bias checklist, with the following categories: (1) publication in peer-reviewed journal, (2) randomisation of treatment or control (3) blinded assessment of outcomes, (4) statement of compliance with regulatory requirements, and (5) statement regarding possible conflict of interest (COI).

\section{Methods of Analysis of EV-Based Treatment effect on Renal Function Decline}

As a principle summary measure in data synthesis, we utilised the standardized mean difference (SMD) with $95 \%$ CI to combine quantitative data where the same outcome was measured using different methods/ scales. SMDs and accompanying variance were calculated for: plasma creatinine, plasma urea, and GFR. To address the issue of outcome dependance due to shared control group, we adjusted the sample sizes by dividing the reported control sample size by the number of included treatment groups (2) to equalise the weight of each group in our meta-analyses. All metaanalyses were performed for random effect models due to no a priori exclusion of studies with different experimental settings. Random-effects models were fitted using restricted maximum likelihood estimation (REML). We fitted a stratified meta-analysis model to evaluate the factors that could mediate the treatment effect. We applied the stratification criteria similar to the previous meta-analysis in CKD by Papazova et al. [16]. These included: 1) model-related factors: (a) animal species, (b) CKD model, (c) CKD etiology due to diabetes, and 2) treatment intervention-related factors: (a) treatment timing, defined as preventive if EV-based therapy was administered before clinical manifestation of CKD (for induced models, between day 0 and day 6 after induction of CKD model; for knock-out models, before clinical manifestation of disease), (b) EV dose (single vs. multiple), and (c) EV origin (allogenic vs. xenogenic). The I-squared index was primarily used to quantify the dispersion of effect size percentage of variability attributable to heterogeneity in a meta-analysis and has justified the application of the random-effects model to produce a combined therapeutic effect across heterogeneous studies. Funnel plots were used to visually assess publication study bias. In a case of visual asymmetry, the presence of small-study bias was examined using the Egger regression-based test. The trim and fill procedure was applied to adjust the results to putative publication bias. All statistical analyses were carried out with the use of Stata software version 16.1 (Stata MP, College Station, TX).

\section{Methods of Analysis of Vesicle-Enclosed miRNAs - Target Prediction, Data Filtering and Visualisation as Interaction Network}

We have investigated the molecules encapsulated in EVs mediating their protective impact, by conducting a bioinformatic analysis. To identify targets of analysed miRNAs we have used IPA software and target scan function with the respective filters: (1) kidney tissue, and (2) an observed or predicted (with high or moderate confidence) interaction. We have build an miRNA-target network, and than subjected the targets to a network analysis. A target-target interaction network was constructed and visualised using Cytoscape software. Gene-gene interaction data were retrieved from String using StringApp package version 1.4.2 for Cytoscape. The EV microRNA database (http://bioinfo. 
life.hust.edu.cn/EVmiRNA/\#!/) was searched for data regarding miRNA abundance in EVs from different sources.

\section{Results}

\section{Search Results and Study Characteristics}

The flowchart of study selection for systematic review and meta-analysis is shown in Fig. 1. The initial search identified 506 articles from PubMed, 306 from Web of Science, and 19 from Cochrane. Four articles were obtained from additional searches. Among 53 full-text articles assessed for eligibility, 35 studies were included in the systematic review, including 11 studies regarding Unilateral Ureteral Obstruction (UUO), 9 studies regarding diabetic CKD settings, 7 studies regarding hypertensive (HT) CKD settings, 5 studies regarding toxic CKD, 2 studies regarding nephrectomy $(\mathrm{Nx})$, and one study in Alport Syndrome. The most frequently used source of EVs were mesenchymal stem cells (MSC) derived from bone marrow, adipose tissue, or umbilical cord. EVs were also isolated from other stem cell (SC) sources, including urine, amniotic fluid (AFSC), and liver (HLSC). Other sources used to derive EVs were: cardiac progenitor cells (CPC), endothelial progenitor cells (EPC), embryonic kidney cells, and STC-like cells. In most studies, EVs were injected intravenously after the CKD model was induced.

The details of the design of the included articles (and records within), classified according to the utilised CKD model (UUO, HT, diabetes and other) is summarised in Table 1, while the complexity of the therapeutic interventions between different CKD settings is illustrated in Fig. 2. The included studies showed considerable heterogeneity with respect to quality and experimental design, f.ex. CKD model, EV origin, time-points of EV administration and dose.

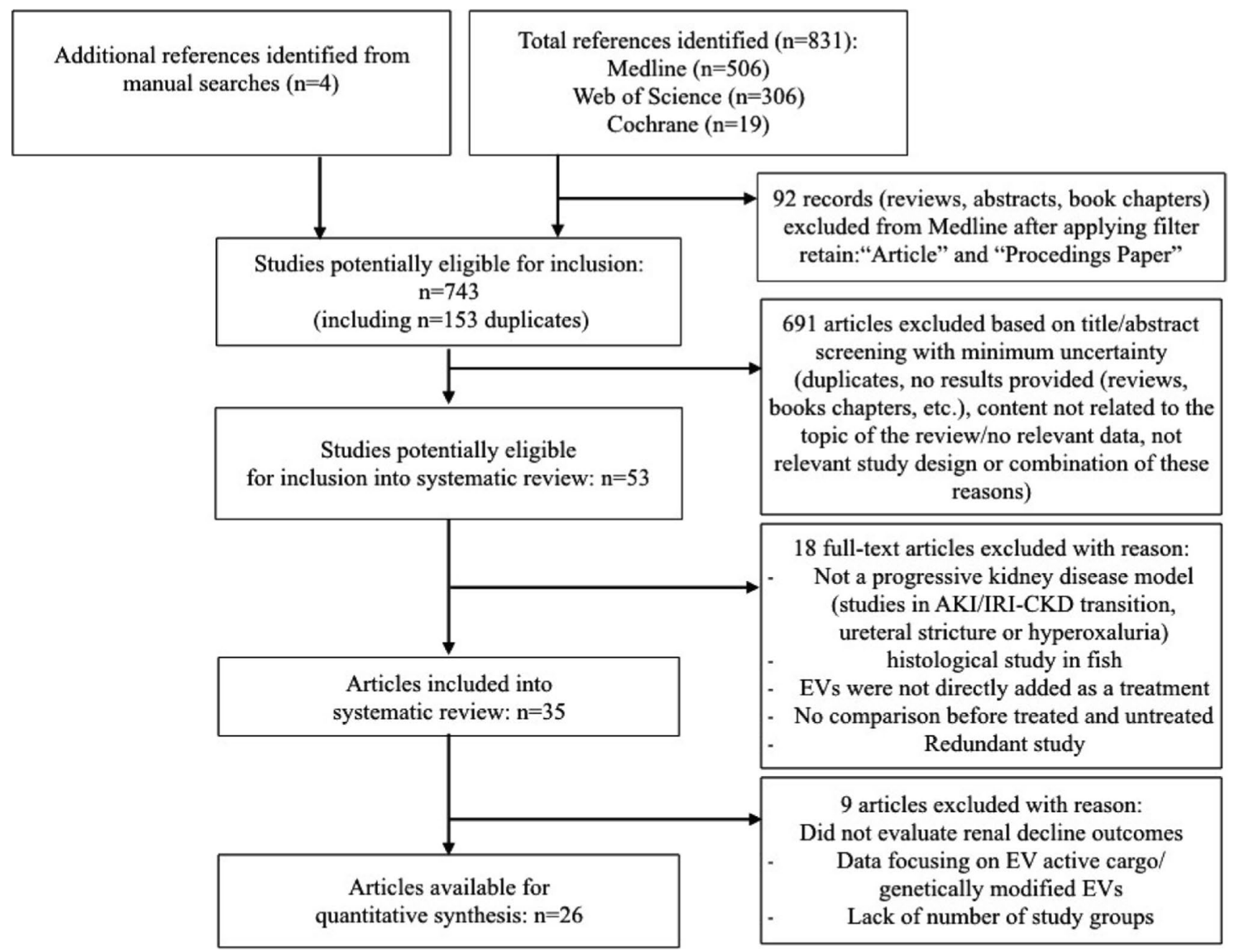

Fig. 1 Flow chart illustrating search strategy and inclusion and exclusion of studies for systematic review and meta-analysis. Articles were selected according to criteria defined in the methods section 


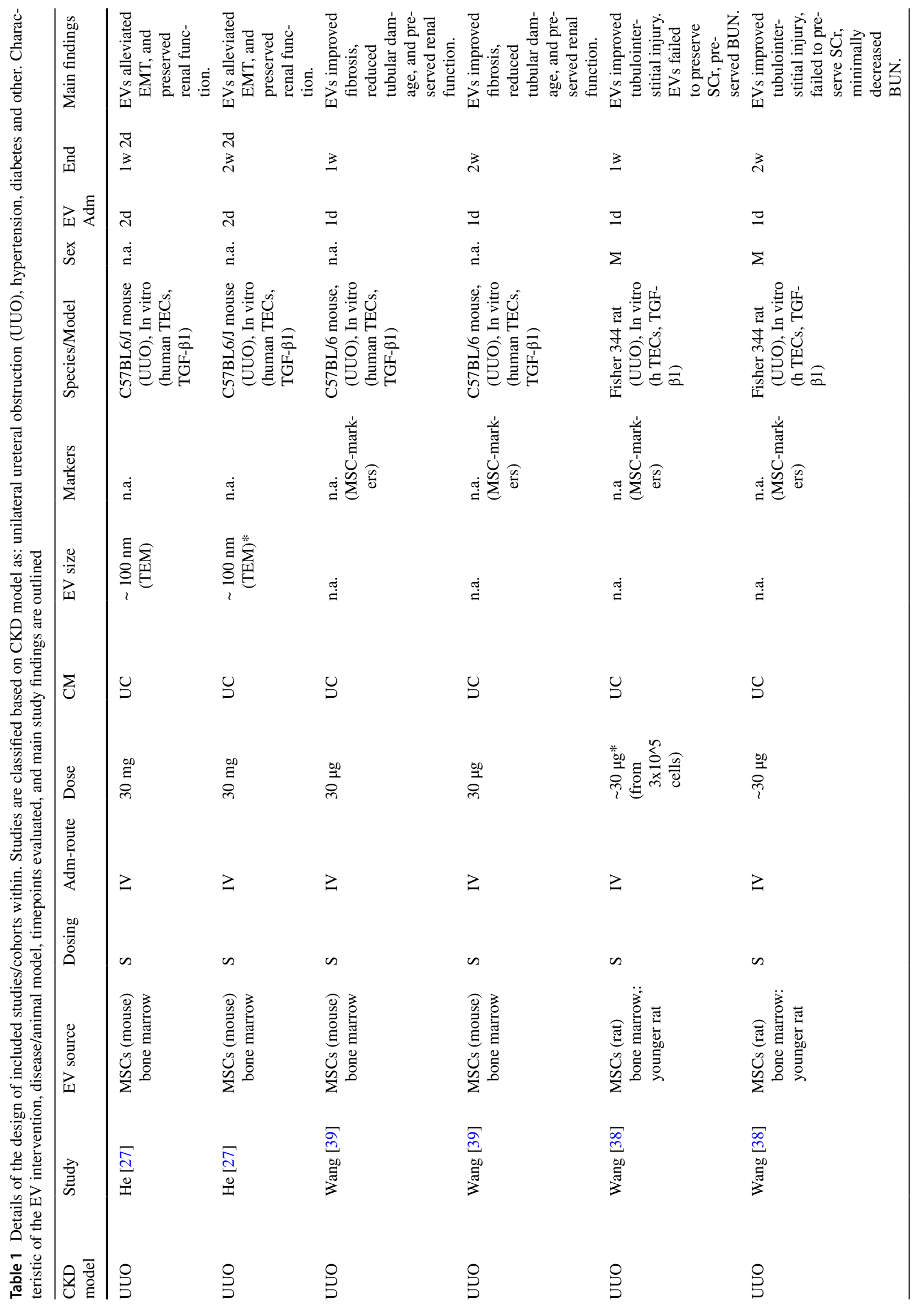




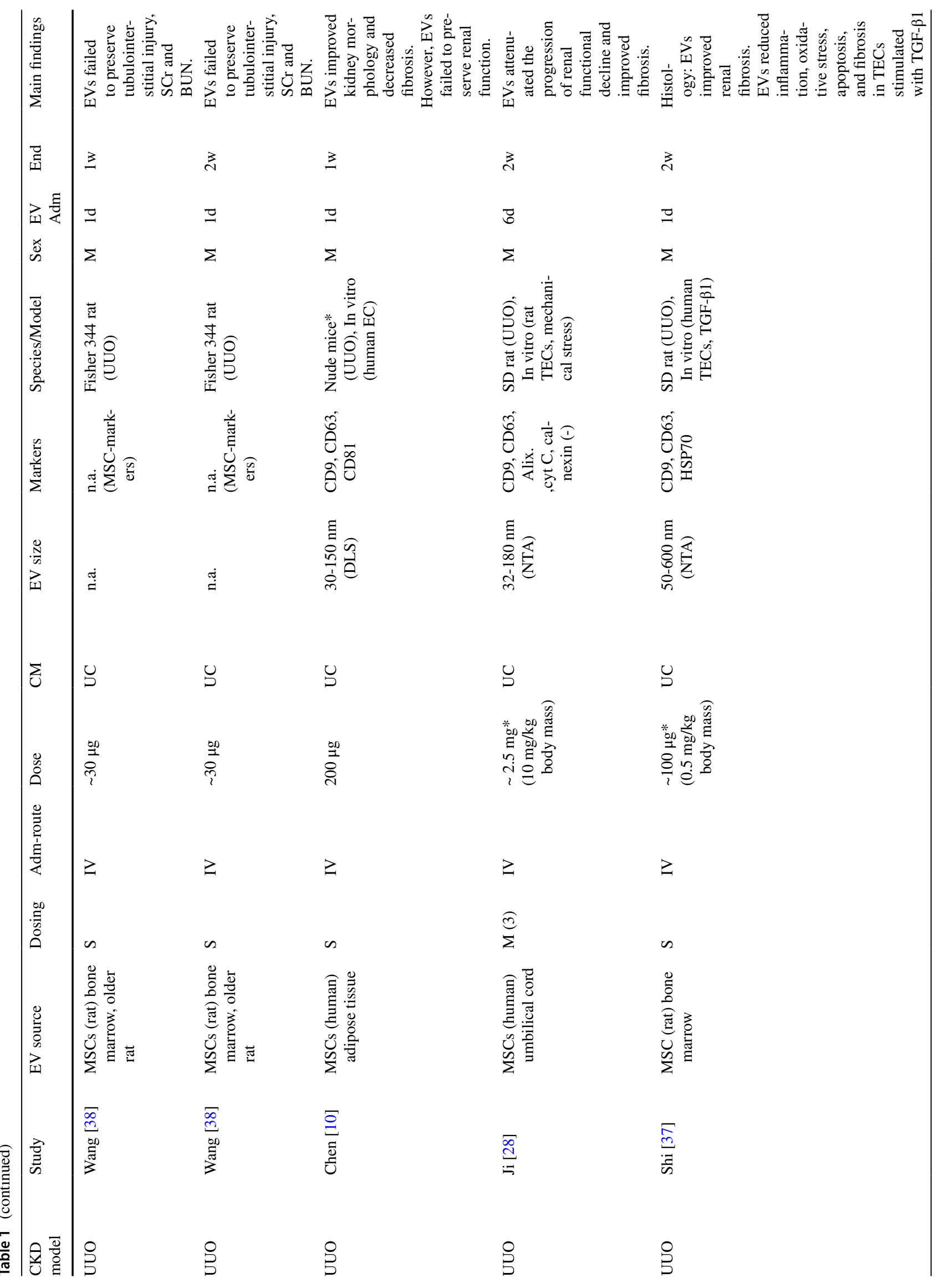




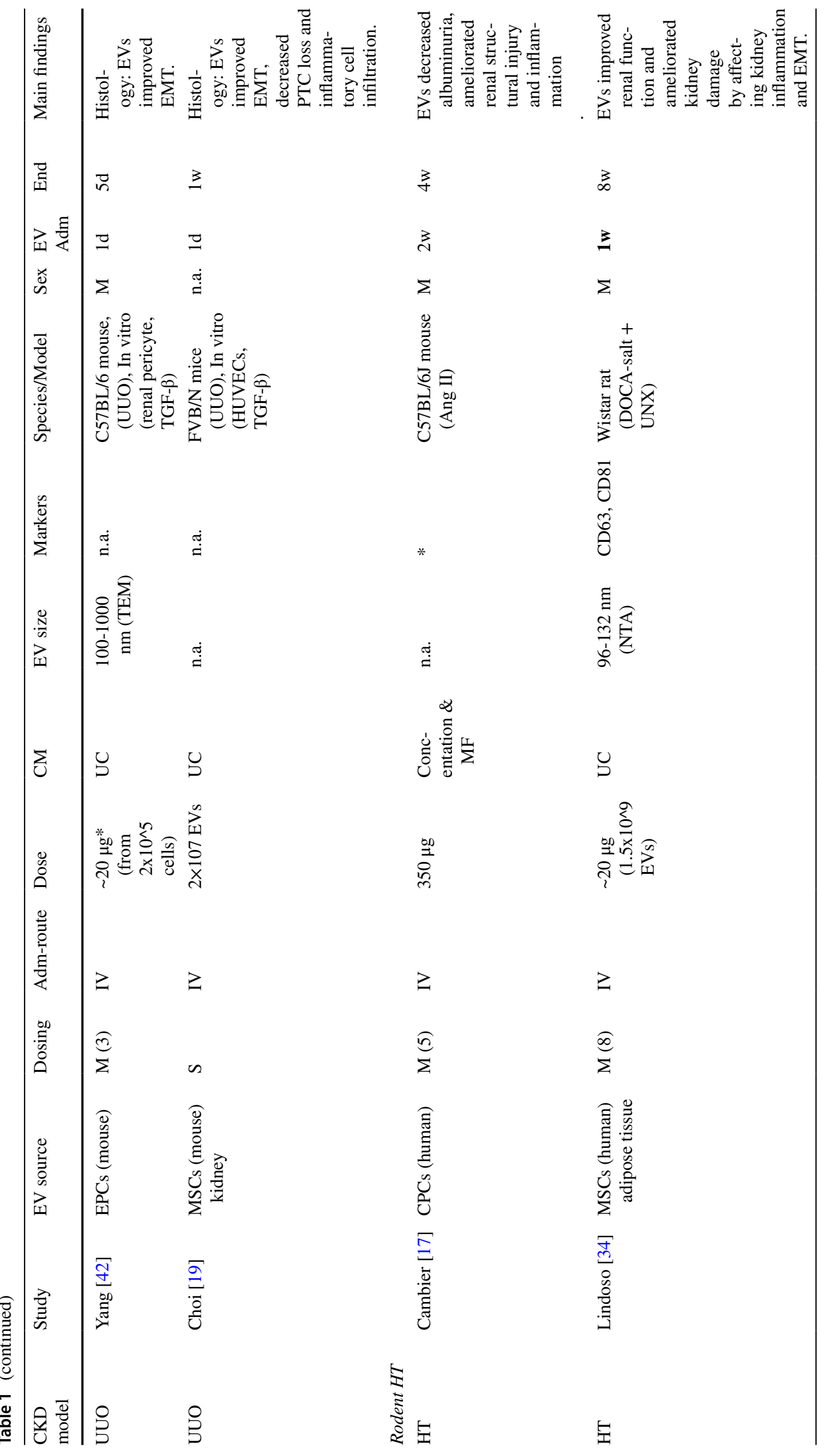




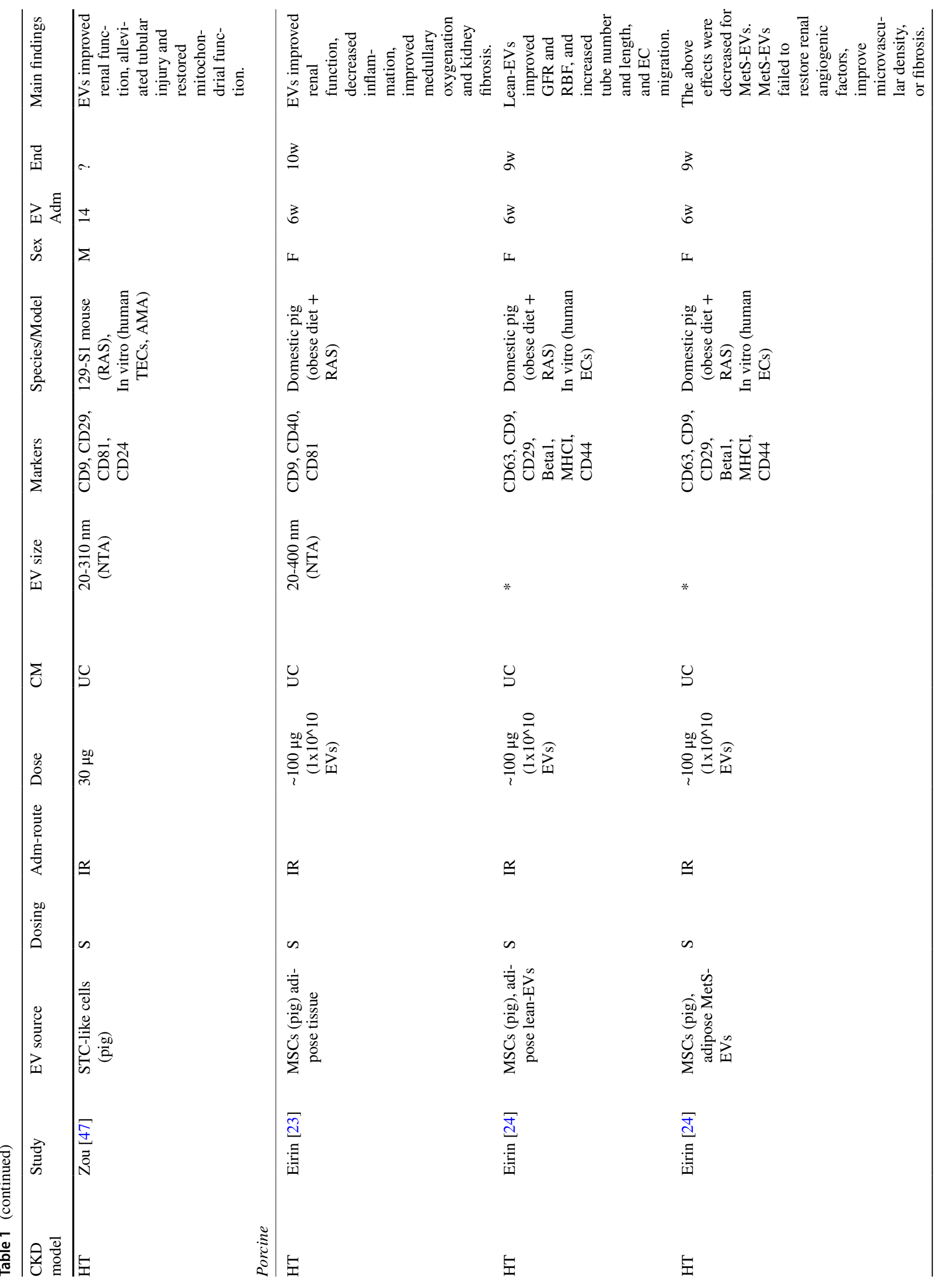




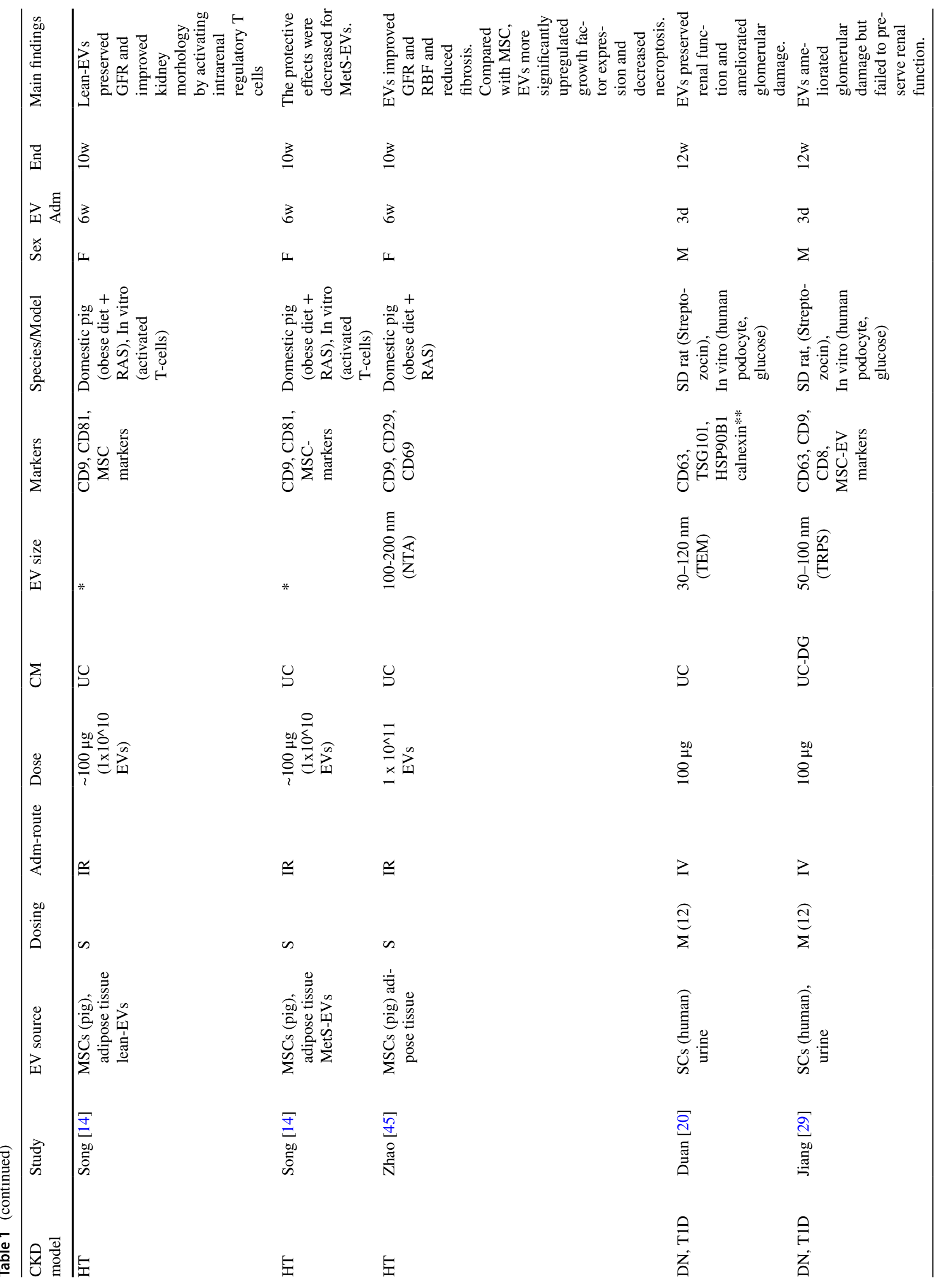




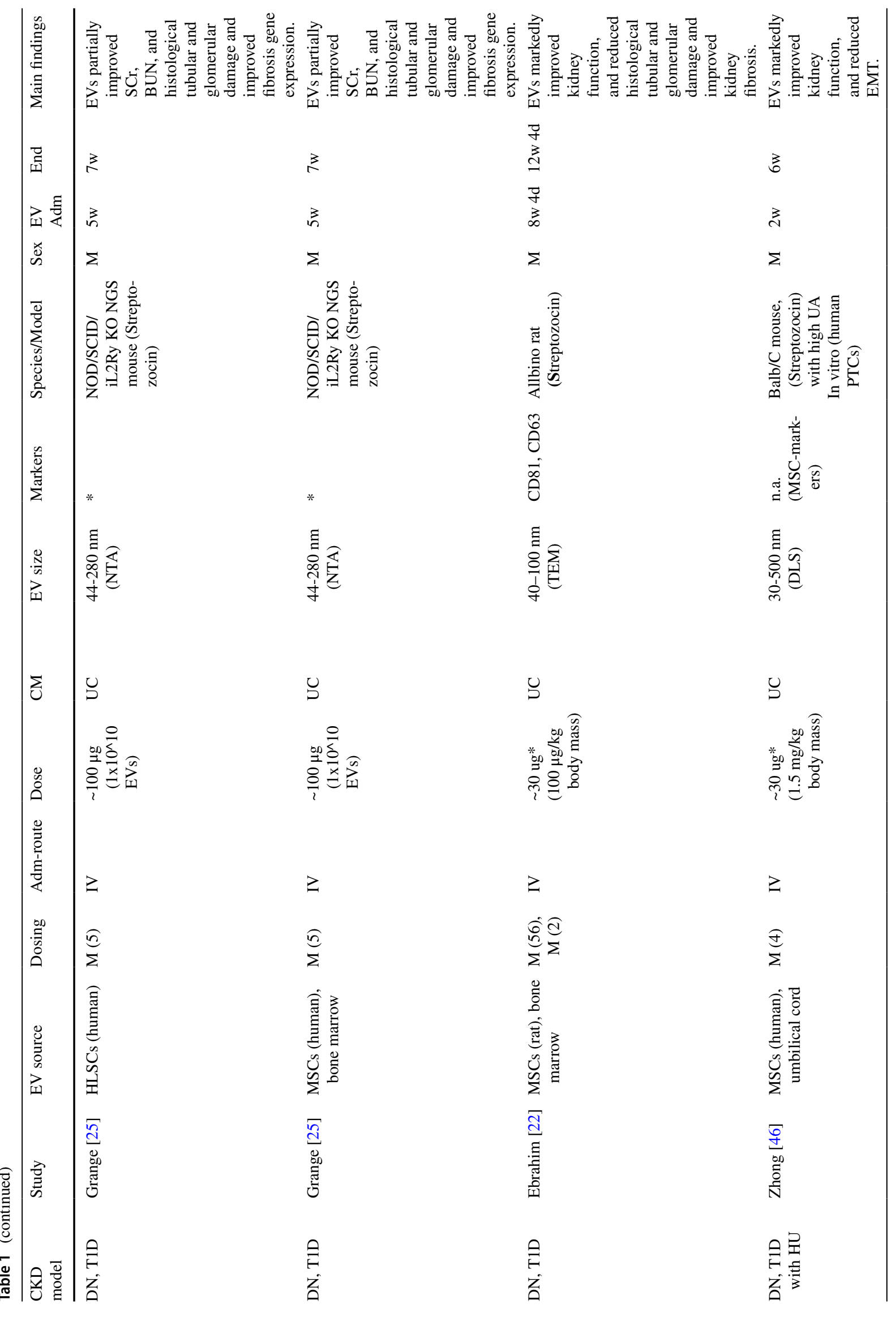




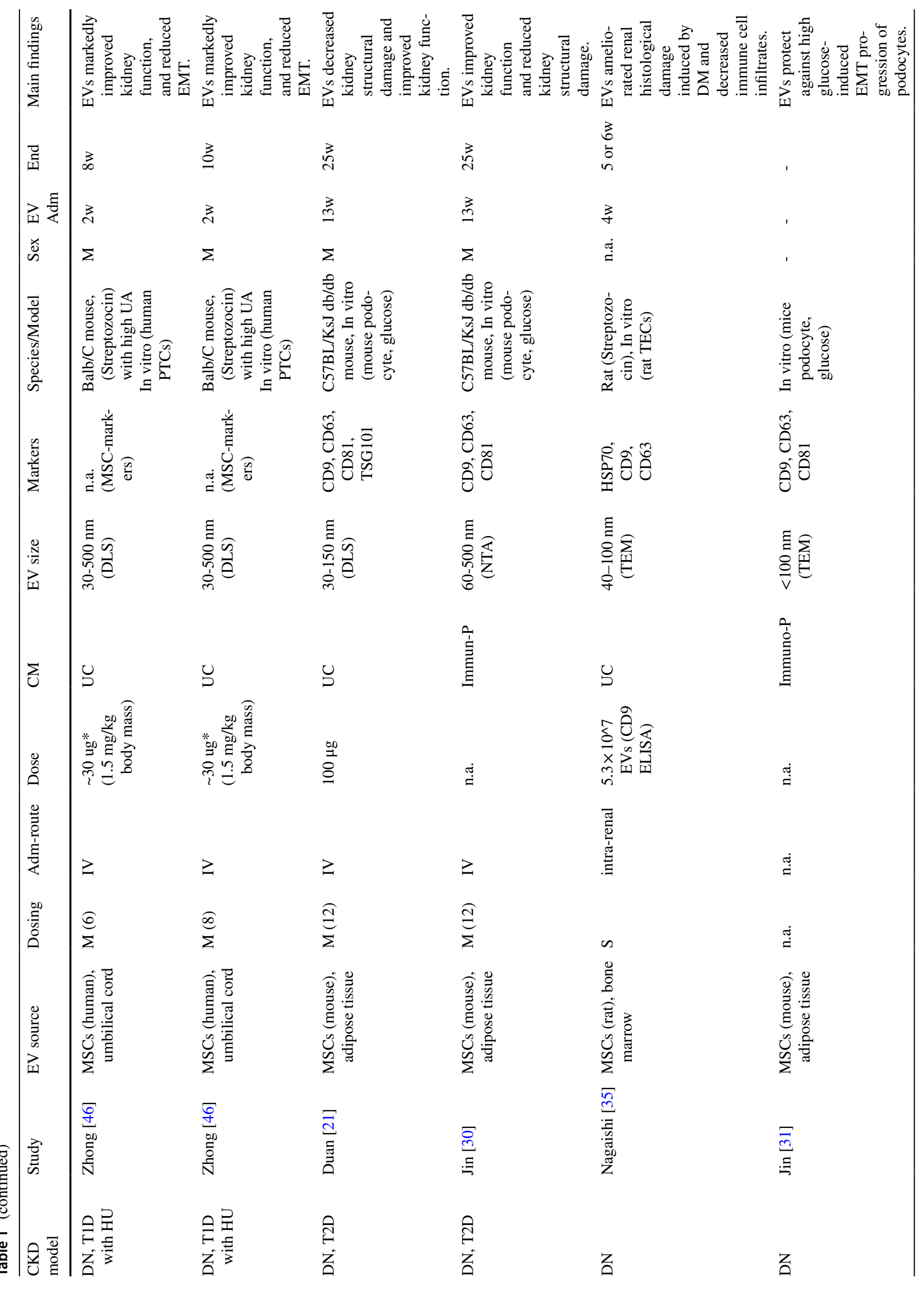




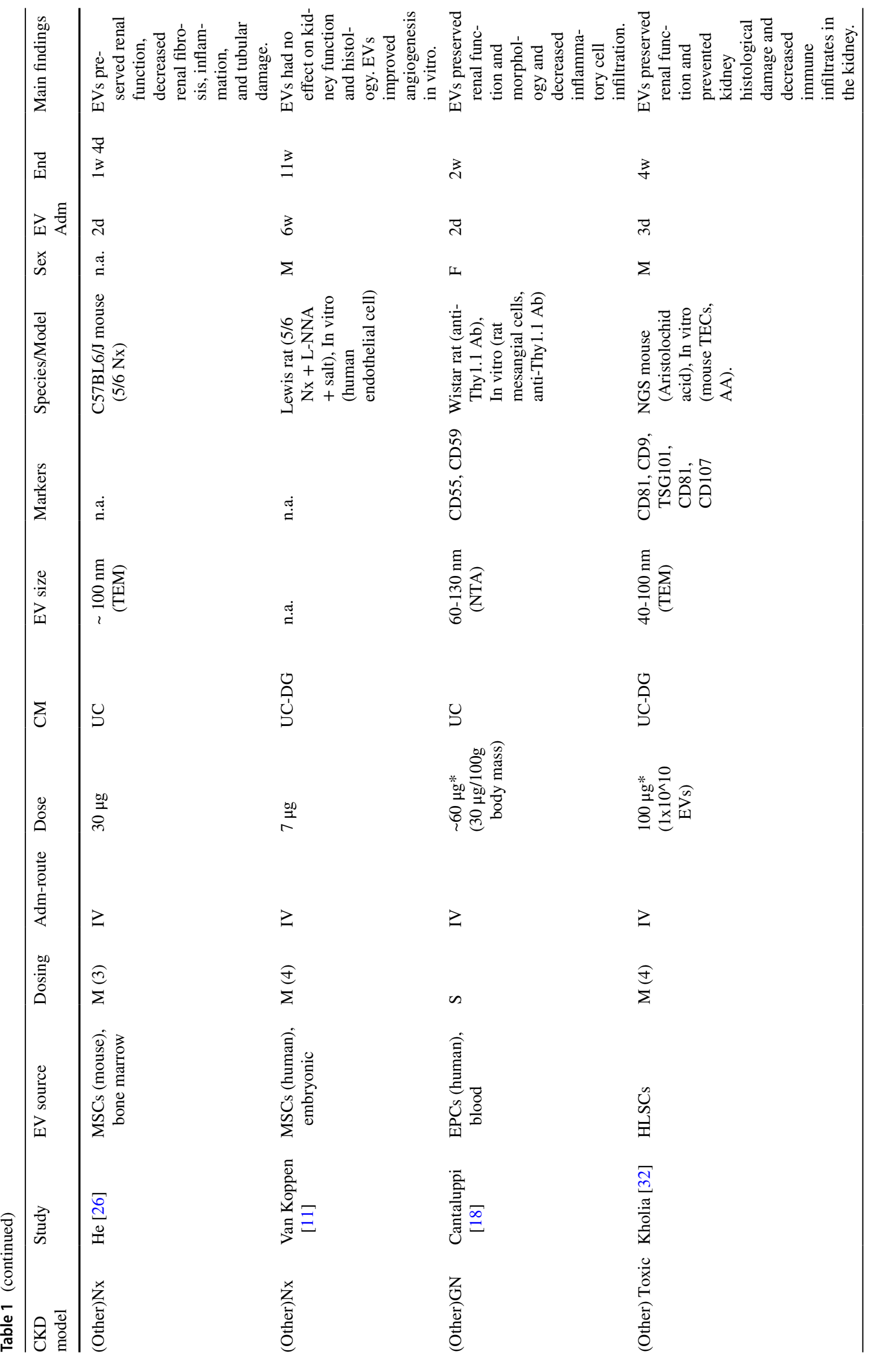




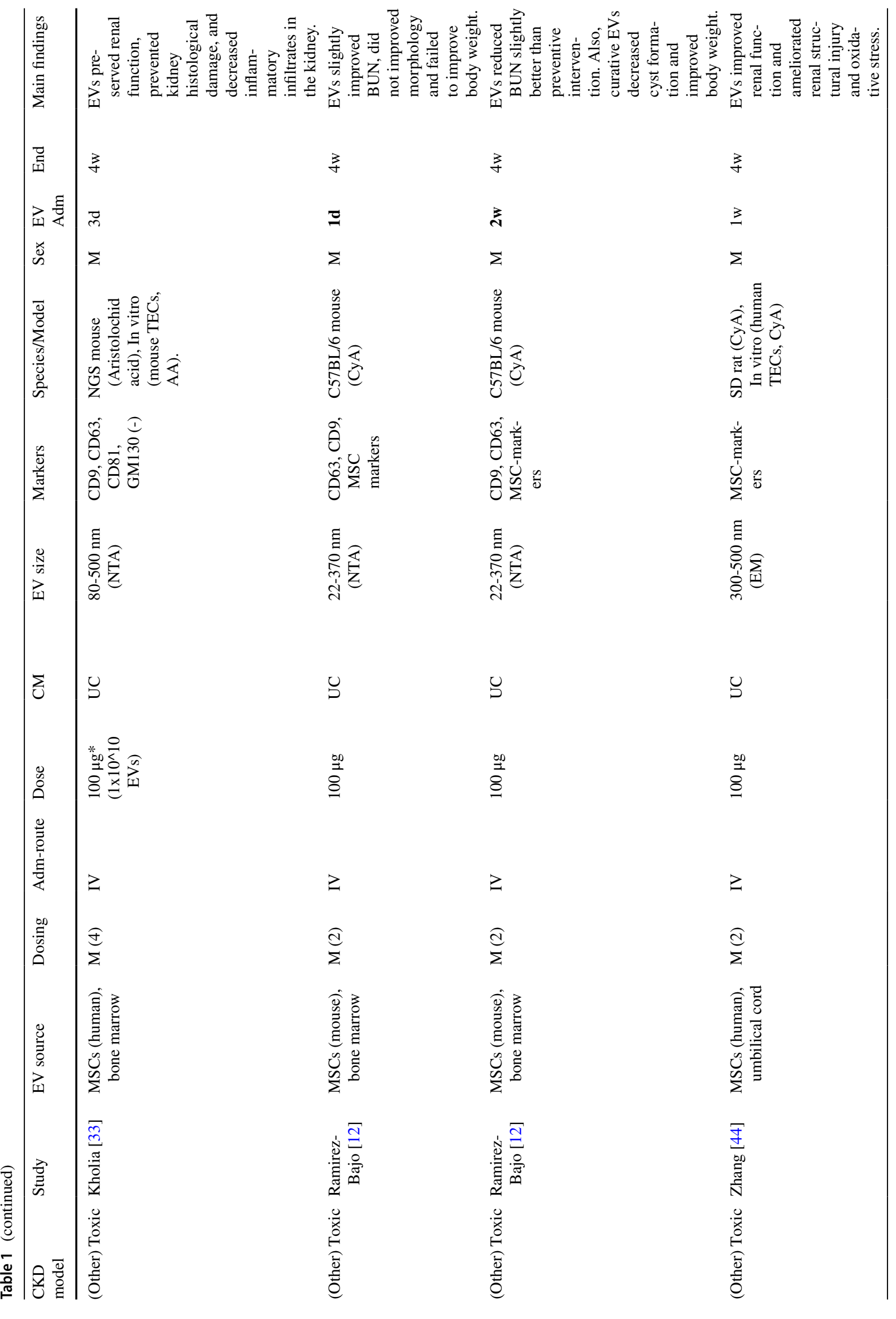




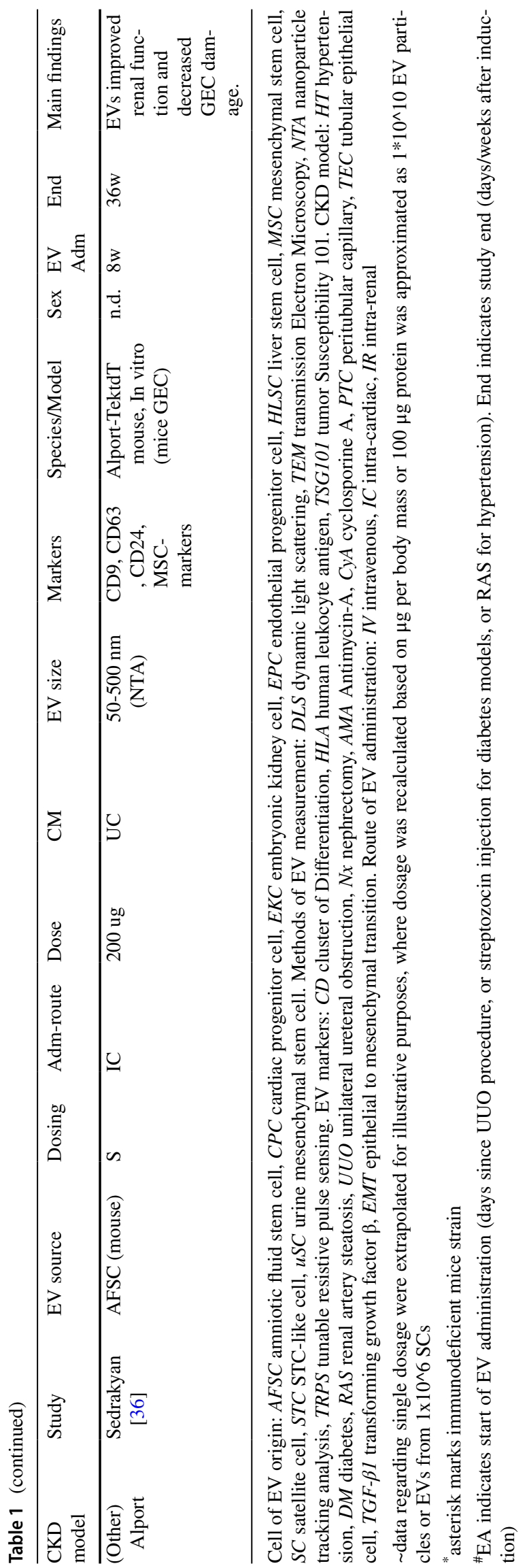

Studies in the UUO model (except one study [42] on EPCs) tested MSC-derived EVs, have utilized single therapeutic doses (ranging from $30 \mu \mathrm{g}$ to milligrams of $\mathrm{EV}$ protein per animal), that were administered (intravenously) briefly (1 or 2days) after model induction (preventive treatments). One study (Chen et al. 2019) was performed in immune-deficient NGS mice and administered human adipose tissue MSCderived EVs [10]. The study by Wang [38] has compared therapeutic effect between preventive administration of smaller doses of bone marrow MCSC-EVs isolated from young adult rats versus administration of EVs isolated from older rats. Only one study [28] has administrated 3, larger doses of EVs (single dose of approximately $2 \mathrm{mg}$ protein per animal), starting from the 6th day after UUO surgery. Importantly, the studies in UUO varied regarding study quality and adherence to ISEV guidelines (Table 1 and Supplementary Table 1).

In contrast to the UUO model, studies in diabetes have utilized multiple doses of EVs that were mainly administered to animals with kidney disease (curative treatment). There were many different (multiple) doses of SCs-EVs, which are summarized in Table 1. Two studies in type 1 diabetes $[20,29]$ have administered urine SC-derived EVs (12 doses) briefly ( 3 days) after streptozocin injection (preventive treatment). The study by Grange et al. have administered curative treatments ( 5 doses of EVs) derived from either human bone marrow MSCs or HLSCs to immunodeficient NGS (NOD/ SCID) streptozocin-induced mice. Another study in type 1 diabetes model (Ebrachim et al., 2018) has injected different (curative) doses of MSC-EVs into Albino rats. Also, one study (Zhong et al., 2019) has administered various doses of umbilical cord MSC-EVs (4, 6, or 8 doses) to a preselected subset of Streptozocin-induced type 1 diabetes mice with high uric acid concentrations [46]. Two studies were performed in type 2 diabetes $(\mathrm{db} / \mathrm{db})$ mouse model $[21,30]$ and injected multiple doses of adipose tissue MSC-derived EVs (12 doses, administered weakly), since the 13th week of age. Additionally, one study in diabetes [35] has utilized intra-renal MSC-derived EV injection of smaller EV doses, and documented histological findings.

Studies in hypertension were conducted in either porcine or rodent CKD models. Studies in porcine CKD (model of metabolic syndrome and renal artery stenosis) utilized single doses of adipose tissue MSC-EVs that were injected 6 weeks after RAS into the renal artery [14, 23, 24, 45]. Two of these studies have utilized MSC-EVs obtained from pigs with metabolic syndrome and from lean pigs [14, 23]. Conversely, two studies in rodent models of HT tested venous injection multiple EV doses. The first study, by Cambier et al., administered 5 doses (350 $\mu \mathrm{g}$ each) of smaller EVs derived from human CPCs, in a mouse model of cardiac hypertrophy and kidney injury. These EVs were administered retro-orbitally, after 2 weeks of chronic infusion of Angiotensin II. The second study, by Lindoso et al., injected 8 smaller doses of human adipose tissue MSC-derived 


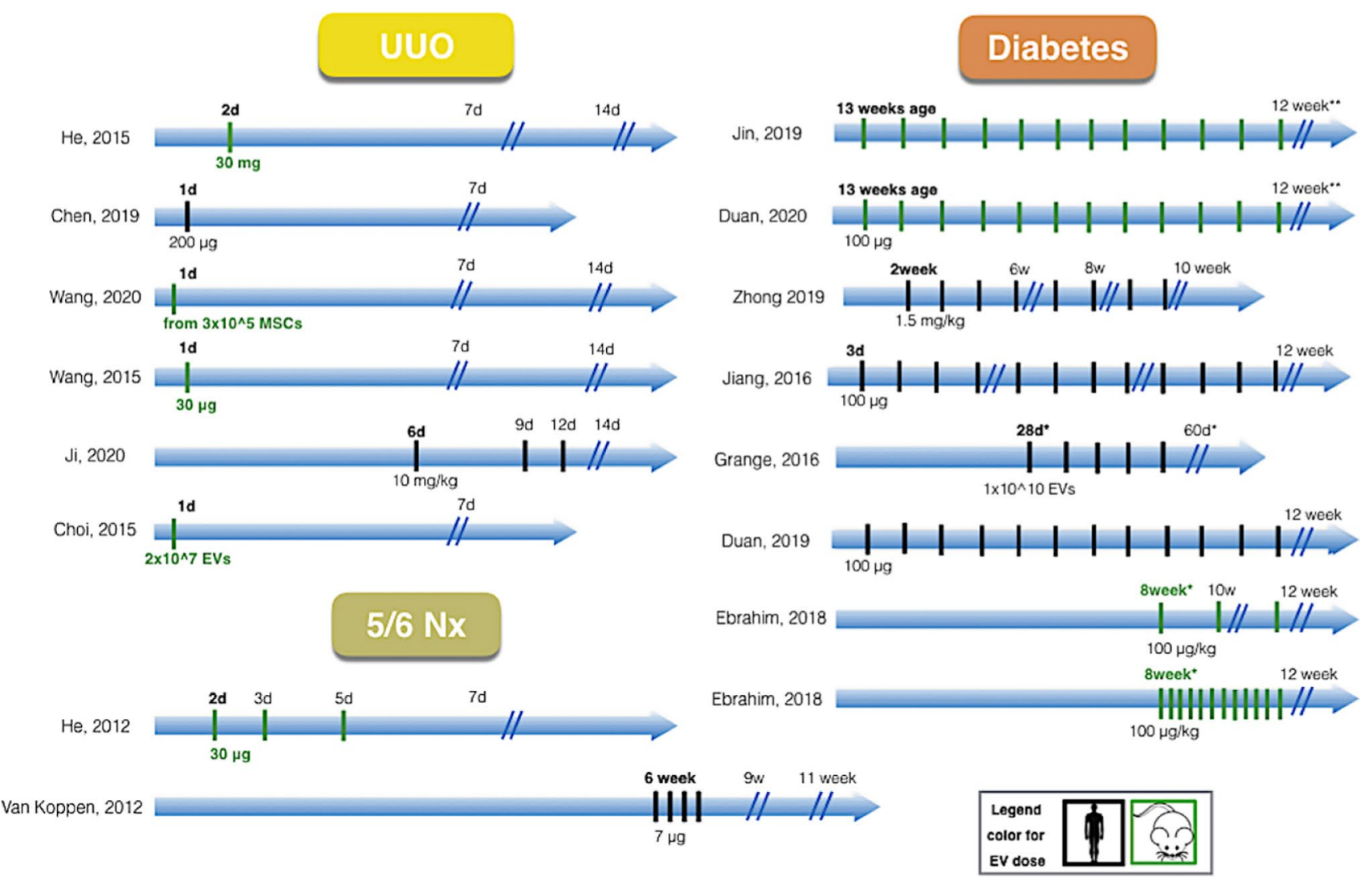

Fig. 2 A roadmap of studies investigating extracellular vesicle (EV)protective effect in animal CKD models. The figure shows studies in obstructive (UUO), nephrectomy (Nx) and diabetic settings of CKD, with $\mathrm{x}$ axis depicting time until the termination of the study. Colour of the doses represents organism of origin of EVs: black, human;

EVs, 2 weeks after nephrectomy (1 week after administration of DOCA-salt). The third study, by Zou [48] injected single doses of porcine STC-like cell-derived EVs into the stenotic kidneys of RAS mice.

All studies in toxic CKD were performed in rodent models and utilized multiple EVs doses. The two studies (by Kholia et al.) were performed in an immunodeficient NGS mouse model, that was induced by injection of aristolochic acid. In these studies, human HLSC or human bone marrow MSC-derived EVs were injected intravenously, with the first $\mathrm{EV}$ dose administered briefly (3 days) after toxicant injection. Another study by Ramirez-Bajo utilized either preventive (the first EV-treatment dose administered 1 day after cyclosporine A administration) or curative character of mouse bone marrow MSC-EV administration (first EVtreatment dose administered 2 weeks after cyclosporine A administration). Another study by Zhang et al. [44] administered human umbilical cord MSC-derived EVs one week after administration of cyclosporine A.

Only two studies were performed in 5/6 Nephrectomy and both of these studies tested smaller EV doses. One study green, mouse, rat. Considerable heterogeneity was visible across the included studies in terms of the experimental models used, time of $\mathrm{EV}$ administration, and number of EV-treatment doses Ebrahim et al. measured renal function after 2 and 4 weeks of EV administration (2 injections per day, since 8 th week after diabetes onset)

He et al. [26] injected 3 doses (30 $\mu \mathrm{g}$ dose) of bone marrow EV-based treatment (preventive treatment). The second study [11] utilized multiple injections of small doses of human embryonic EVs into rats with late CKD stage.

Finally, the studies that focused on genetically modified EVs overexpressing defined miRNAs (or silencing experiments) have been presented separately in Supplementary Table 2.

\section{Quality of Extracellular Vesicle-Research}

The International Society for Extracellular Vesicles had proposed a set of standards to characterise EVs preparations for preclinical studies. They include adherence to EV extraction and characterisation protocols, appearance in transmission electron microscopy (TEM), paired with counting of EVs with particle enumeration methods, expression of the "EVenriched" markers, or the absence of expression of non-EV components [48]. In line with those guidelines, we assessed the quality of the included studies by investigating the EVspecific experiments. Differential ultracentrifugation (UC) was the most commonly used EV separation technique, 
while other methods, such as density gradient-UC [12, 32], polymer-based precipitation [21, 40], and immunoaffinity capture [30,31], were used by $5-15 \%$ of studies. To improve the specificity of EV separation, 7 studies had utilised complementary techniques following the primary step, such as ultrafiltration, or application of density gradients $[13,17$, $20,25,29,30,33,41]$.

The studies documented that the morphology of the preparations was consistent with the characteristics of EVs by providing vesicle characteristics (most commonly TEM and NTA results), and by measuring the EV enriched markers. In case of several studies, the ISEV criteria have been met in their previous publications. Together, most authors (60\%) provided characterization of vesicles with two complementary techniques. Positive EV markers were measured by $80 \%$ of the authors; however, these markers were mostly limited to transmembrane proteins. Also, the number of the reported markers varied between studies. Two studies have supplemented those findings with negative markers to exclude the presence of larger types of EV. Importantly, while reviewing the studies it was visible that although in the early phase of $\mathrm{EV}$ research in $\mathrm{CKD}$, many authors did not provide $\mathrm{EV}$ characteristics, the adherence to the ISEV standards is improving gradually with time, which is in the right direction - towards further standardisation of the results, and enabling more precise comparison between the studies.

In a formal bias analysis, we investigated the publication risk of bias using the selected items from the CAMARADES checklist [49]. The included studies were published in impacted journals, provided statements regarding compliance with regulatory requirements, and conflict of interest. Randomisation of animals was reported in a half of all studies. Measurements and analysis of histology outcomes was only performed blindly by the authors in one third of the included studies. All the results of the quality assessment at individual study level are summarised in Supplementary Table 1.

\section{Therapeutic Effect of Extracellular Vehicle-Based Therapy on the Kidney Function Decline}

Of all eligible publications examining EV-based therapy in CKD animal, more than $90 \%$ of studies reported significantly improved kidney histology, and more than $80 \%$ of eligible studies confined significantly improved markers of kidney function (Table 1). Also, some publications observed significant protective effect on renal structure, although no statistically significant changes in ether creatinine or eGFR/ urea were detected $[11,29]$, or observed inconsistent or borderline changes [13, 38]. Only one study in nephrecomized rats reported the lack of protective effect on either kidney function or the structural measurements [12]. However, this particular study has utilised smaller EV doses.
There were 26 studies eligible for meta-analysis of renal decline outcomes: 19 studies (total 27 comparisons) that measured plasma creatinine concentrations, 15 studies (total 25 comparisons) that measured plasma urea concentrations, and 7 studies (total 9 comparisons) that measured glomerular filtration rate (GFR) (See Fig. 1).

As main approach to evaluate the combined EV therapeutic effect (on plasma creatinine and urea), we have performed the meta-analyses for all records, excluding the studies that did not report any EV characteristics (EV-enriched markers, or EV size/visualisation with TEM), and studies performed in large animal (porcine) models (see Fig. 3). As additional approach, we have performed sensitivity analyses for all the eligible studies, to evaluate the impact of these exclusions on the overall findings (Supplementary Fig. 3). The study design and time-points for the individual study cohorts within creatinine and urea analyses are characterised in Fig. 3 and Supplementary Fig. 3. The GFR analysis was mostly done in hypertension and contained studies that reported creatinine clearance ( 3 studies, all in rats) and studies with a multidetector computed tomography (4 studies, all in pigs).

The treatment of animals with EVs has consistently improved ( $p$-values $<0.001$ for all 3 outcomes) all three parameters, supporting the potential protective properties in CKD (Fig. 3, and Supplementary Figs. 1-3). The graphical illustration of the treatment effect of EV-based therapy on plasma creatinine and plasma urea based on CKD model is shown in Fig. 3. The SMDs for both markers were considerably different between CKD models (Fig. 3A and B). For plasma creatinine the SMDs were: -2.06 (95\% CI: -4.46; 0.35) for UUO, -2.97 (95\% CI: -5.31; -0.63) for hypertension (HT)induced CKD, -8.97 (95\% CI: -16.41; -1.53) for diabetesinduced CKD, -1.65 (95\% CI: $-2.42 ;-0.86$ ) for toxin-induced CKD, and -2.04 (95\% CI: -3.23 ; -0.85 ) for $5 / 6$ nephrectomy (Fig. 3A). For plasma urea the SMDs were -3.06 (95\% CI: $-6.02 ;-0.10)$ for UUO, -4.52 (95\% CI: $-7.73 ;-1.32$ ) for diabetes-induced CKD, -1.25 (95\% CI: $-2.24 ;-0.25$ ) for toxic CKD, and -0.63 (95\% CI: $-1.61 ; 0.35$ ) for 5/6 nephrectomy (Fig. 3b). The subsequent analysis including all study cohorts that reported renal decline is illustrated in Supplementary Fig. 3.

The analysed outcomes showed significant asymmetry of the results in funnel plot analyses indicating a possibility of potential bias. In addition, the results of the Egger regression-based test ( $p$-values $<0.001$ for all 3 outcomes) supported the existence of small publication bias. The trim and fill procedure identified 4 missing studies for creatinine, and 1 missing study for urea, however did not influence the overall findings for either outcome measures. Statistical heterogeneity was high in all analyses (with I-squared index exceeding 75\%), and we have conducted a stratified meta-analysis to investigate potential sources of heterogeneity in creatinine and urea concentrations. 
A

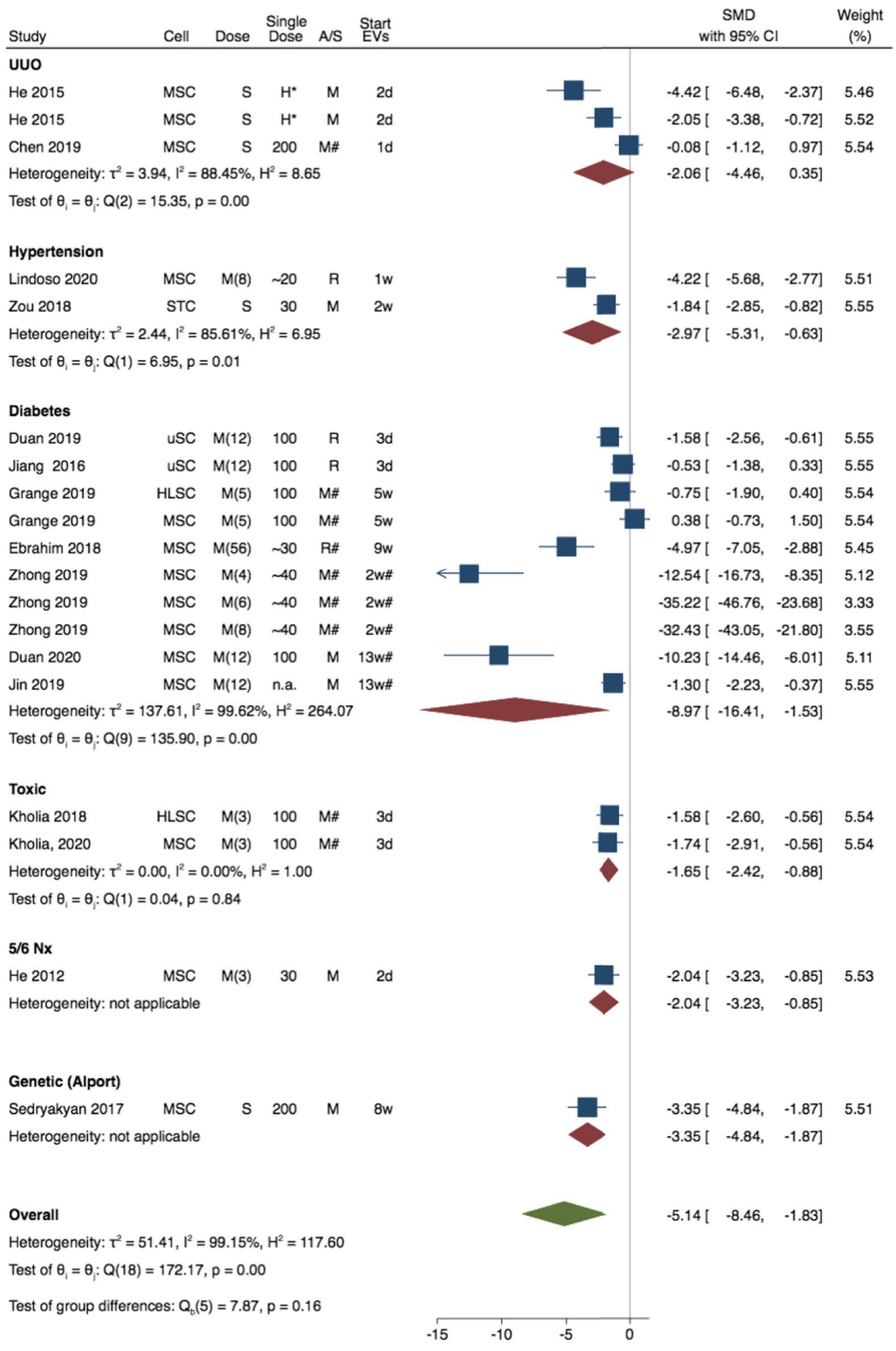

Random-effects REML model

Fig. 3 Forest plot for the therapeutic effect of extracellular vesicle (EV)-treatment on renal function decline in experimental CKD: Unilateral ureteral obstruction, hypertension, diabetes, toxic-CKD, and nephrectomy (Nx) models. Data represent SMD in (A) plasma creatinine and (B) plasma urea calculated for treaded versus non-treated comparisons of all records, excluding large animals (porcine models) and studies that did not report EV characteristics (Analyses for all study cohort is show in Supplementary Fig. 3). Abbreviations: 95\% confidence interval $(95 \% \mathrm{CI})$. RE, random effect. Cell indicates cell of EV origin, dose indicates approximate (recalculated as in Table 1) single dose in $\mu \mathrm{g}$ protein, $\mathrm{A} / \mathrm{S}$ indicates animal species, start $\mathrm{EV}$ theraphy indicates days or weeks since model induction 
B

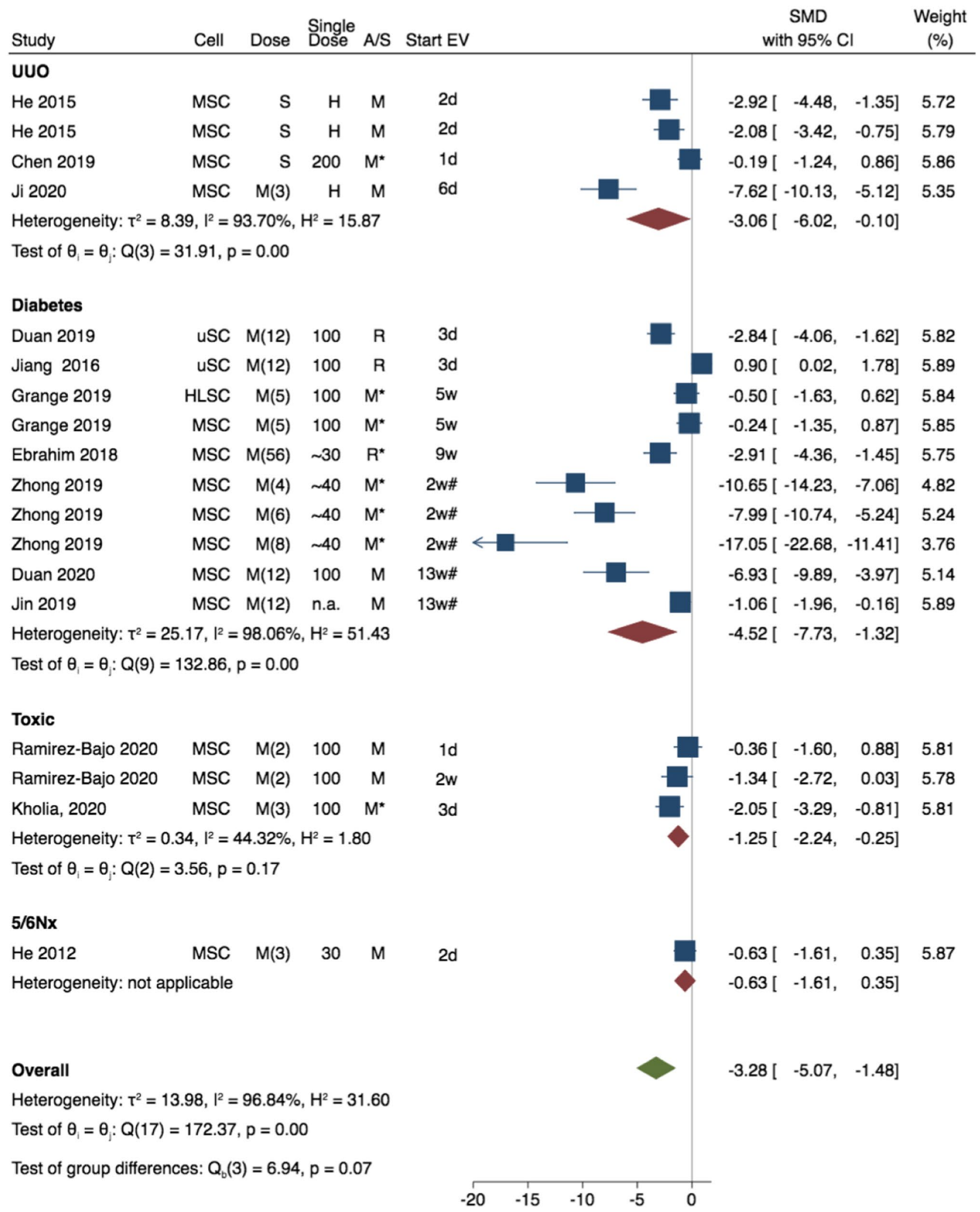

Random-effects REML model

Sorted by: order

Fig. 3 (continued) 
The stratified analyses for plasma creatinine indicated that timing of the therapy (curative vs preventive), and administered dose of the EVs were potential determinants of the therapy efficacy (Supplementary Fig. 2A). Considerably higher treatment effect was observed in diabetic $(-8.97$ (95\% CI: $-16.41 ;-1.53))$ than in non-diabetic $(-2.24(95 \%$ CI: $-3.10 ;-1.38)$ ) models of CKD (p-value for the difference $=0.06)$. The treatment effect was -6.72 (95\% CI: -11.77 ; $-1.67)$ in animals receiving multiple doses of EVs in comparison to -2.21 (95\% CI: $-3.60 ;-0.81)$ in animals receiving single dose of EVs (p-value for the difference $=0.09$ ). The treatment effect was -8.68 (95\% CI: $-15.19 ;-2.17)$ in animals receiving curative treatment in comparison to -1.56 (95\% CI: -2.28 ; -0.85) for animals receiving preventive treatment ( $\mathrm{p}$-value for the difference $=0.03$ ).

The directions of the observed differences in urea concentration between subgroups of diabetes-induced CKD versus other CKD models were similar to the pattern observed for creatinine concentrations, albeit not significant (SMD -4.52 (95\% CI: -7.73; -1.32) vs SMD - 1.97 (95\% CI: $-3.40 ;-0.54)$; p-value for the difference $=0.15$ ). Also, there was a trend towards better treatment response in urea for multiple EV doses $((-3.68(-5.87 ;-1.48)$ vs -1.65 $(-3.26,-0.04) ; \mathrm{p}$-value for the difference $=0.1))$, and later time-point of EV administration ((-5.00 (95\% CI: -8.43; $-1.56)$ vs -1.82 (95\% CI: -3.27 ; -0.37$)$; p-value for the difference $=0.09$ ). However, the number of included studies was relatively low and CIs were wide, indicating low statistical power to detect significant differences. The SMDs in plasma urea were -3.42 (95\% CI: -5.64; -1.19) in mice, and -3.00 (95\% CI: -6.32; 0.35) in rats (p-value for the difference $=0.83$ ). There were no significant differences in creatinine or urea reduction by EV source (xenogeneic vs. allogenic); however, statistical power was also low.

To evaluate the underlying differences in the effectiveness of EV-treatment we analysed their biological effect across the included studies. We assessed the histological findings, biochemical data, as well as active molecules contained within EVs, and their involvement in CKD pathways. In order to illustrate the biological implications of vesicular miRNAs in the kidney we performed interaction network analysis between the target genes of the miRNAs identified based on the literature search. In the following section, we catalogue these findings according to the mechanism underlying the $\mathrm{EV}$ protective effect and the CKD model.

\section{Mechanisms of Action of Extracellular Vesicles and Enclosed Molecules According to the Chronic Kidney Disease Model}

A primary component, that is common to all CKD aetiologies, is the progressive fibrosis of kidney cells leading to impaired renal function. Thus, one of the main features to consider when assessing the EV nephroprotective capacity involved modulating fibrosis-related mechanisms (decreasing inflammation, decreasing cell damage and inducing angiogenesis, improving anti-ROS response), and treating fibrotic process by regulating the expression of pro-fibrotic programs of kidney cells. To induce the anti-fibrotic protective response, EVs were derived either from stem cells (SCs), kidney cells, or were generated from engineered cells, by using lentiviral RNA transfer. The cell types that were utilised as EV source, based on their involvement in CKD mechanism, are summarised in Supplementary Table 3 and represented in Fig. 4.

Twenty-five of the eligible studies showed that EVs ameliorated kidney histology: 13 studies reported reduced glomerulosclerosis (GS) and 23 studies reported reduced tubulointerstitial fibrosis (IF). Also, 26 studies supplemented the histological findings with the decreased expression of pro-fibrotic mediators (Supplementary Table 2). In the early studies in experimental CKD, He et al. investigated nephroprotective properties of MSC-EVs in animal models of Nx and UUO, showing ameliorated functional damage and reduced fibrosis. [26, 27]. MSCs-EVs were as effective as MSCs themselves in accelerating functional recovery from CKD in UUO mice in vivo [27]. These EVs inhibited TGF$\beta 1$-induced morphological changes in mice tubular epithelial cells in vitro, and these effects were attributed to their carried miRNA cargo [27]. Similarly, Choi et al. reported that MSC-EVs reversed morphological changes induced by TGF- $\beta 1$, ameliorated peritubular capillary rarefaction, and reduced IF in UUO mice [19]. In line with these findings, MSC-EVs were subsequently reported to reduce GS and/ or TF in the settings of UUO [11, 28, 39, 41], hypertension $[15,23,24,45]$, and diabetic CKD [22, 25, 35]; where the damage was improved via mechanisms involving RNA or protein transfer. Among the studies that attributed the protective properties of MSC-EVs to their miRNA content, 3 studies performed microarray profiling inside MSCEVs - the predicted targets of the vesicular miRNAs were implicated in extra-cellular matrix and collagen synthesis and degradation [25, 27, 39]. Further, Zhong et al. demonstrated that EVs ameliorated IF in STZ-induced diabetic mice [46] via modulation of the kidney expression of P15 and P19 molecules (leading to an improved cell cycle arrest) by EV-enclosed miR-451a. Jin et al. found that coincubation of MSC-EVs with podocytes treated with glucose decreased EMT progression via regulation of ZEB2 transcription factor through EV-encapsulated miR-215-5p [31]. Three studies in animal UUO administered engineered EVs that over-express protective miRNAs capable of an anti-fibrosis effect. First, the study by Wang reported that the addition of let7c-MSCEVs repressed collagen type IV $\alpha 1, \alpha-S M A$, and TGF- $\beta$ R 1 expression in rat TECs that had been exposed to TGF- $\beta 1$ [40]. Next, miR-26a-HEK-derived EVs were reported to suppress the TGF- $\beta$ signalling pathway in the injured kidney 
by targeting TGF- $\beta 1$ and CTGF [43]. Third study by Wang et al showed that intramuscular injection of miR-29-EVs into UUO mice reduced TGF- $\beta 3$, and decreased interstitial collagen accumulation in the kidney [41], suggesting that EV anti-fibrotic protective effect was exerted through the TGF- $\beta$ singling pathway inhibition. Among studies that attributed the protective anti-fibrotic properties of MSC-EVs to their protein content, the study by Ji et al. performed global EV proteome profiling [28] identifying enrichment of the TGF$\beta 1$, TLR, VEGF, and ubiquitin-related enzymes - CK1 $\delta$ and $\beta$ TRCP. Administration of these MSC-EVs to UUO rats improved fibrosis by regulating Yes-associated protein (YAP), a co-activator of the Hippo pathway. By contrast, the EV protective effect was impaired with EV-CK1 $\delta$ and $\beta$-TRCP knock-down.

Stem cell-EVs were also documented to modulate renal fibrosis through their anti-inflammatory and immunomodulatory effect. The anti-inflammatory effect of EVs was wellassessed in hypertension (HT) and the metabolic-related CKD models. Regarding HT, the immunomodulatory EV effect was attributed to a reduced renal immune infiltration and modulation of functional programming of macrophage populations. Eirin et al. showed that intra-renal administration of MSCEVs containing IL-10 mRNA attenuated kidney inflammation in the MetS/RAS pigs by switching renal macrophages from M1 to M2 phenotype [23]. Consistent with this macrophage reprogramming mechanism, multiple studies showed that EVs reduce the expression of pro-inflammatory mediators (such as TNF- $\alpha$, MCP-1, IL- 6 , and iNOS) and increase the expression of anti-inflammatory mediators (such as IL-10) in the kidney [13, $17,21,24,25,33,37]$ or circulation [15, 17, 24, 45]. Eirin et al demonstrated that the protective effect was diminished when MSC-EVs derived from pigs with metabolic syndrome were used as compared with MSC-EVs from lean pigs [24]. Extending the mode of action of lean MSC-EVs, Song et al. reported that induction of intrarenal regulatory $\mathrm{T}$ cells through TGF- $\beta$ was required for their anti-inflammatory effects [15]. Further extending the mechanisms of MSC-EVs in HT, Lindoso et al. reported down-regulation of EMT by modulating the kidney miRNA signature as well as induction of immunomodulatory mechanisms in EV-treated DOCA-salt rats [34]. EVs administration has led to normalised renal function, blood pressure, inhibited macrophage recruitment, down-regulated the kidney expression of pro-inflammatory mediators (PAI1, MCP-1, IL-6), and decreased the markers of tubular and glomerular injury. Zhao et al. compared the effects of the administration of MSCs and MSCs-EVs into the stenotic kidney [45]. Both therapies demonstrated beneficial effects on fibrosis, inflammation, and microvasculature. However, in this setting, EVs increased the expression of growth factors more effectively than MSCs. This was explained by EV enrichment with miR532-5p, which can modulate Angiopoietin-1. Finally, Cambier
Fig. 4 Regulatory mechanisms of extracellular vesicle-encapsulated content: a representation of the investigated molecules encapsulated in mesenchymal stem cell (MSC)-EVs, amniotic fluid stem cells (AFSC)-EVs, cardiac progenitor cell (CPC)EVs, endothelial progenitor cell (EPC)-EVs, urine stem cell (uSC)-EVs, and kidney STL-like cell-EVs, based on involvement in fibrosis, inflammation, cell damage, and oxidative stress. Details of the study design of the EV effector molecules are presented in Table 2. The figure was created with the use of Biorender

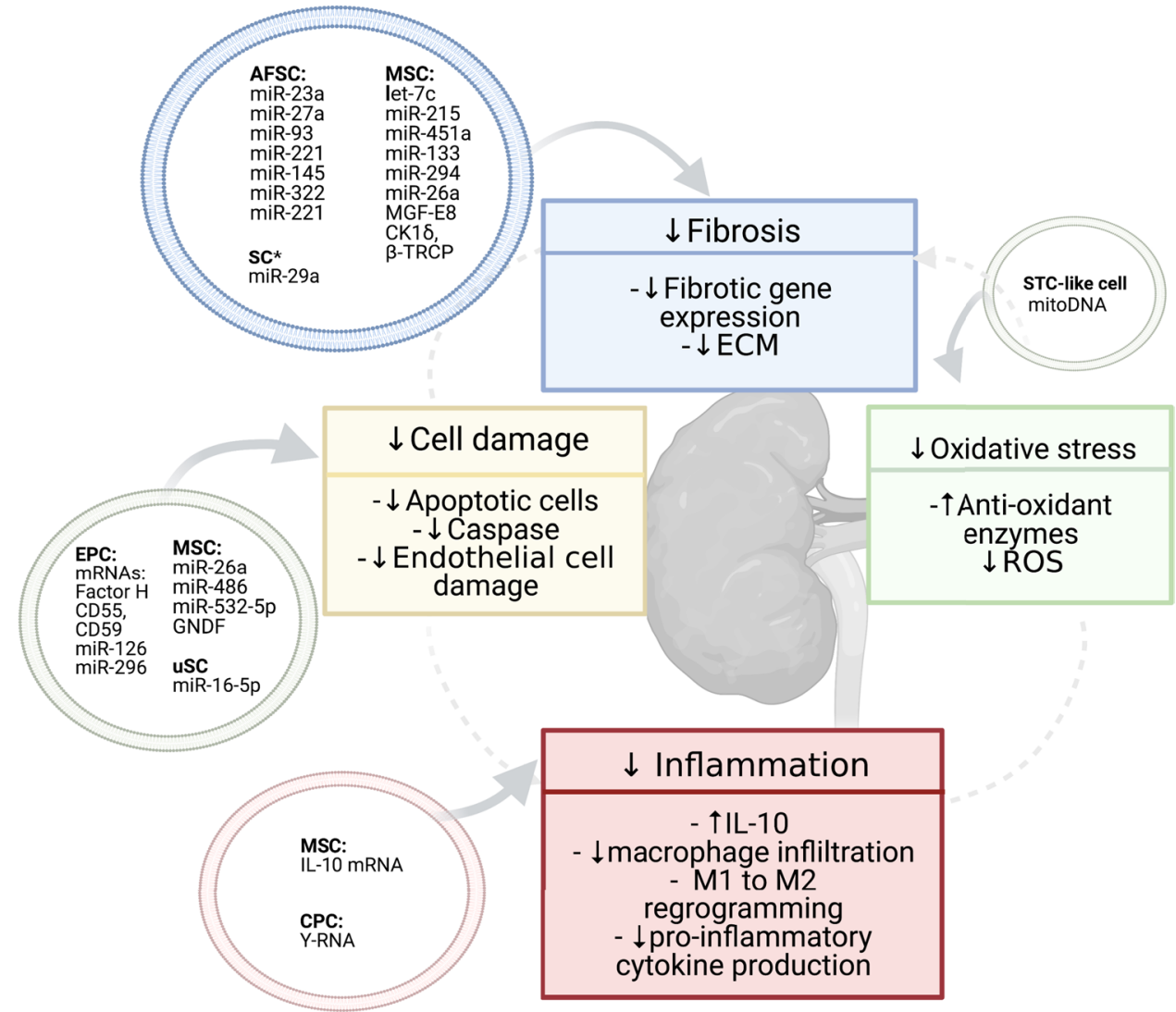




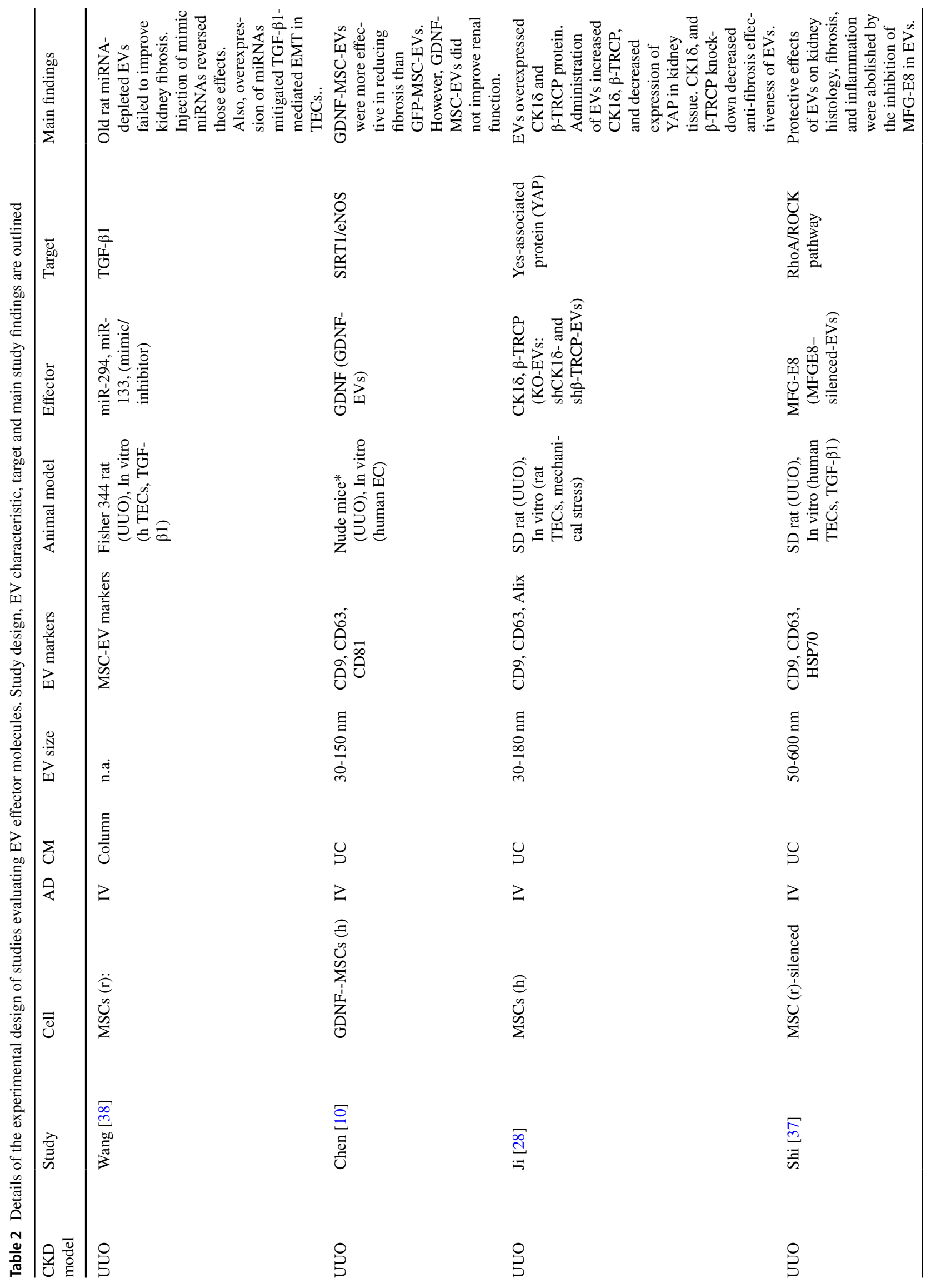




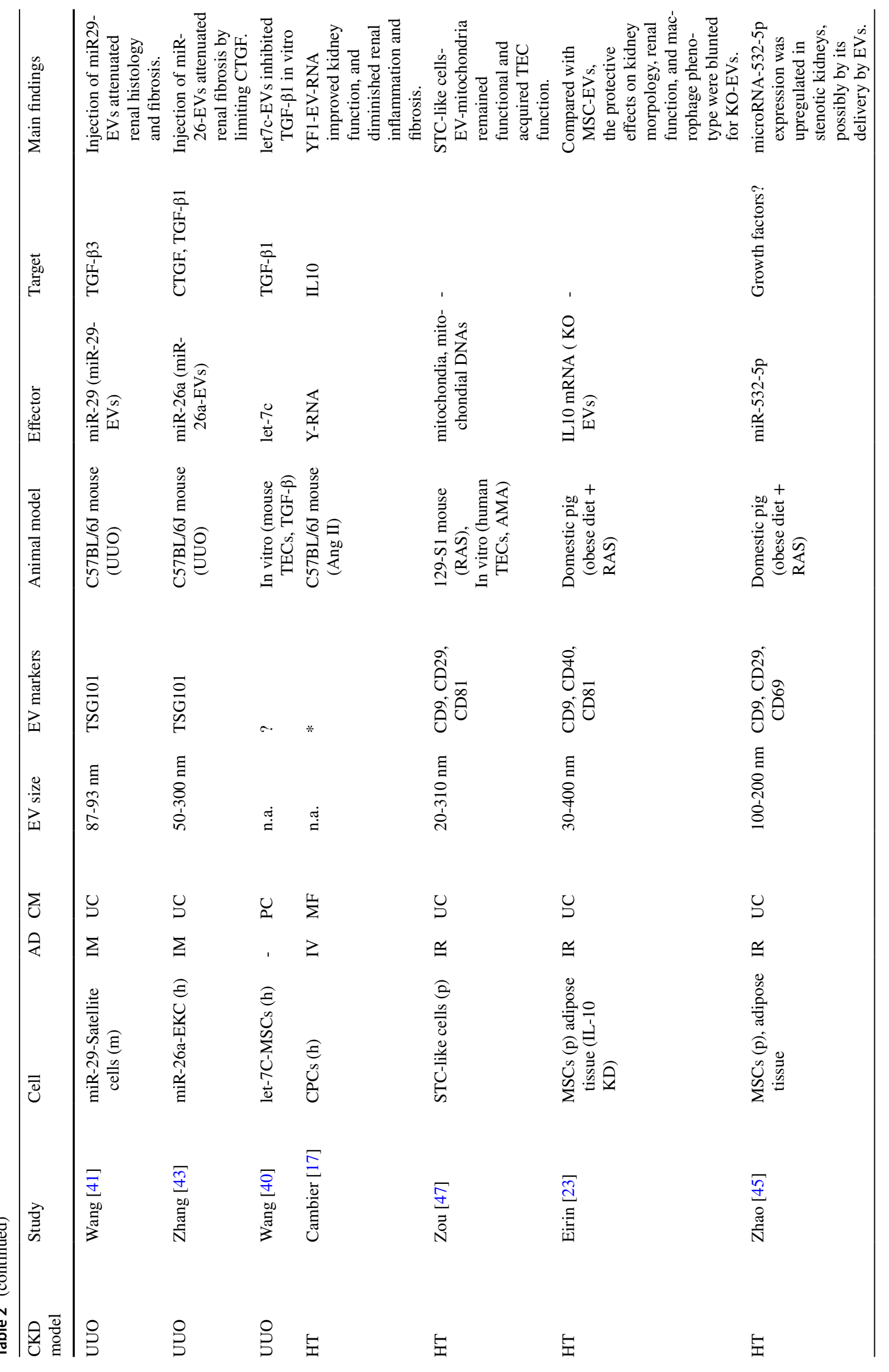




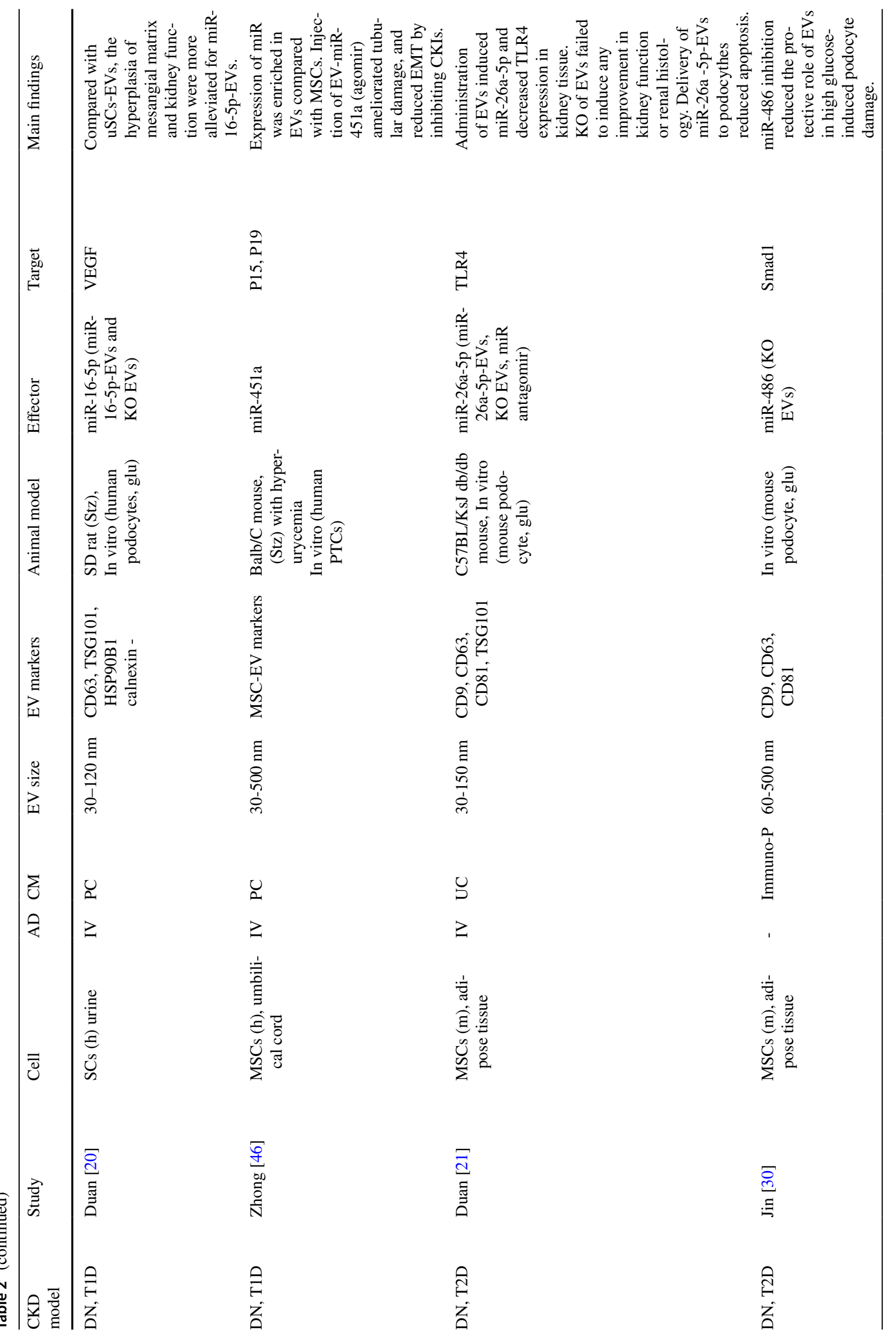




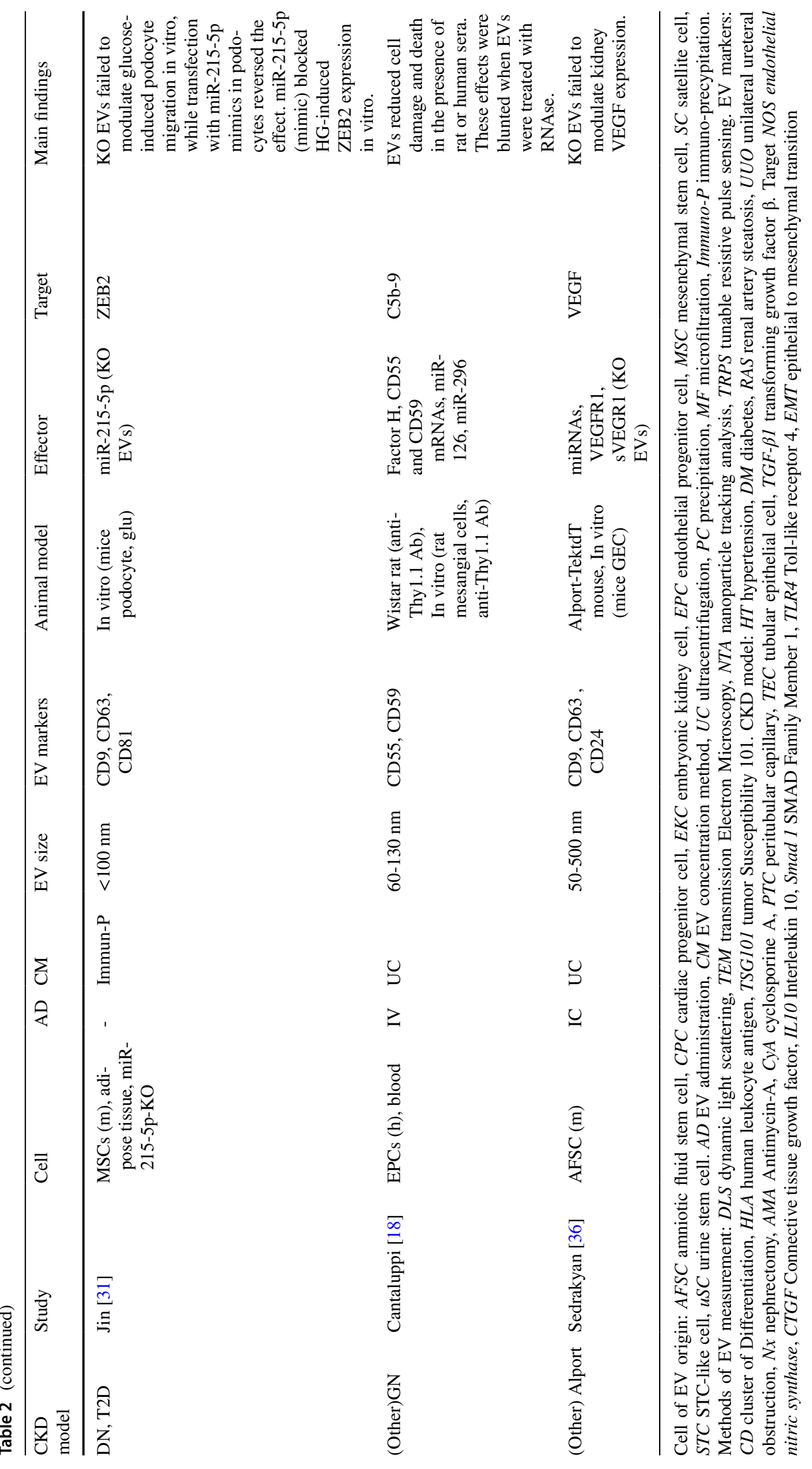


et al. investigated CPC-EVs and YF1 RNA fragment, and reported improved cardiac hypertrophy, kidney function, as well as reduced fibrosis, and diminished inflammation through induced secretion of IL-10 in plasma, heart and kidney [17].

As outlined above, EVs were shown to mediate their their anti-inflammatory nephroprotective effect through interactions with various types cells of innate and adaptive immune system, including macrophages, monocytes and T-cells. Consequently, their anti-inflammatory role might be modulated differently between immunocompetent animals compared with the immunodeficient animals, that lack adaptive immune response. Immunodeficient animal strains (such as NGS, Balb/C, nude) were used by several studies utilizing human EV transplant, in order to avoid xenogeneic EV transplant-induced immune response and toxicity. Two of those studies that were conducted in toxic CKD have performed immunostaining in kidney tissue of NGS mice, to report that the animals receiving EV-based therapy had a significantly lower expression of CD45 positive cells, FSP-1 and $\alpha$-SMA positive myofibroblasts. As these mice lack an adaptive immune system due to their genetic background, the authors concluded that the CD45 (leucocyte common antigen) positive cells were likely to be part of the innate immune system, suggesting that inflammatory responses can be modulated independently of adaptive immunity.

Regarding the diabetic CKD, Grange et al. reported the protective effect of MSC-EVs and HLSC-EVs on structural damage, glomerulosclerosis, and tubulointerstitial fibrosis in a mouse model of diabetes induced by streptozotocin (STZ) [25]. Utilising RNA profiling of the kidney tissue the authors found that SC-EV treatment normalised a significant number of the fibrosis-related and inflammation-related genes that were induced by diabetes, including MMPs, TIMPs, and certain chemokines. Along with changes in renal morphology and the patterns in gene expression, an in silico analyses of the EV-enclosed miRNAs, identified putative EV targets belonging to pro-fibrotic signalling. Likewise, Nagaishi et al. reported the anti-inflammatory, and cell protective effect of MSCs in diabetes, which was modulated through EVs [35]. Those MSC-EVs protected against apoptosis, ameliorated TECs damage, decreased inflammatory infiltrates, and reduced fibrous component of the interstitial space.

Multiple studies investigated EV effect focusing on kidney cell damage/survival pathways, including apoptosis [20, 21, 29, 30, 47], necropoptosis [45], and autophagy [22, 30]. Regarding diabetes-induced CKD, Duan et al. showed

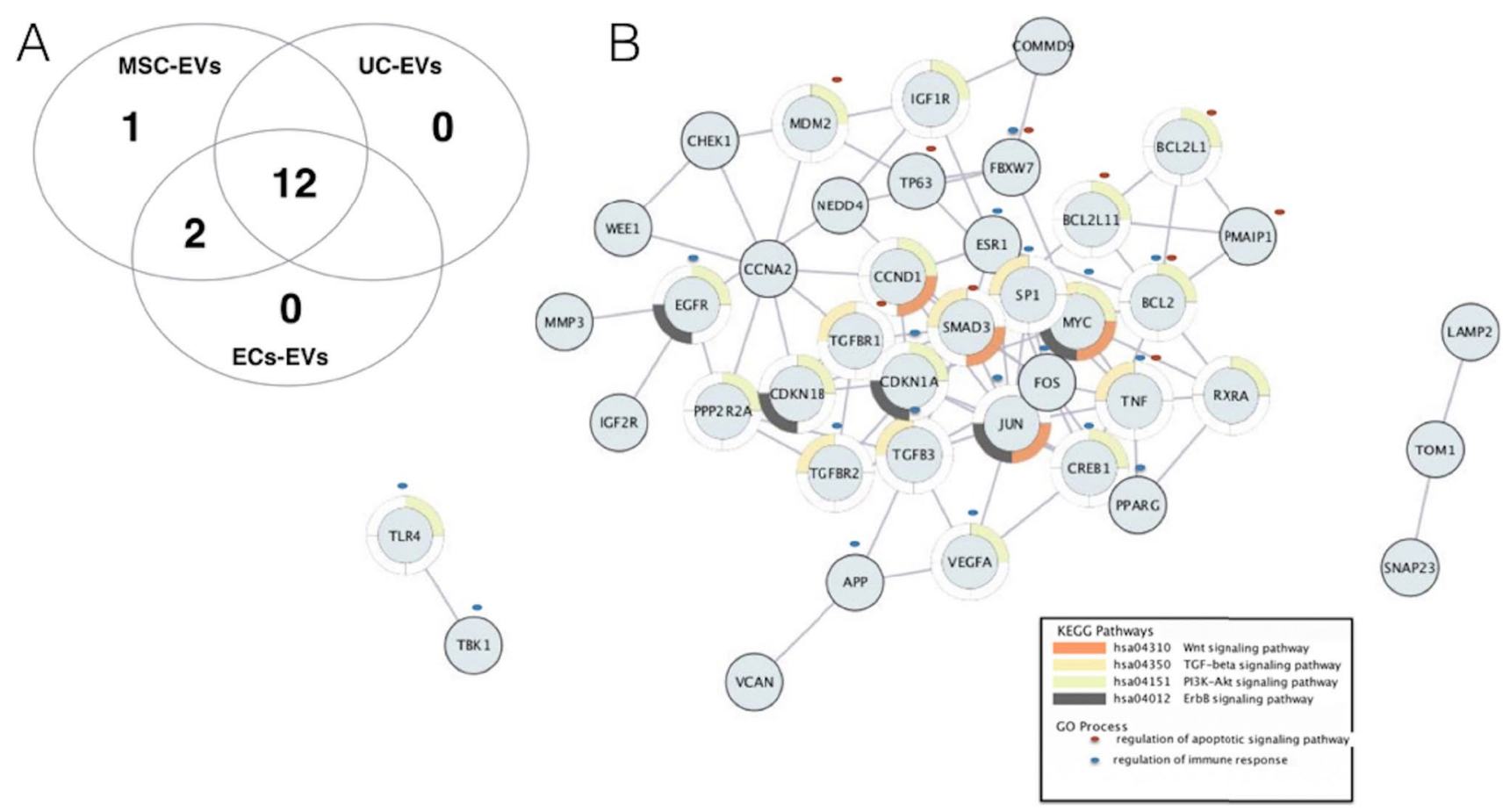

Fig. 5 Regulatory mechanisms of extracellular vesicle-encapsulated (EV) content: bioinformatic analysis of protective miRNAs: (A) Venn diagram illustrating an overlap of the protective miRNAs in EVs from different cell sources. The miRNA expression data were obtained from the EV miRNA database at http://bioinfo.life.hust.edu. cn/EVmiRNA): only miRNAs with expression above $100 \mathrm{rpm}$ threshold were included. (B) miRNA Target-target interaction network.
The nodes indicate target genes, and the edges represent interactions. Only the interactions with a high evidence, ie. evidence score above 0.7 were retained. The molecules without interactions in the PPI network were removed from the figure. Colour in a circle represents different KEGG pathways implicated in kidney fibrosis. Blue, and purple marks represent specific GO process - regulation of immune response, and cell death, respectively 
that SC-EVs promoted podocyte survival in studies utilising rodent models of T1D and T2D [20, 21]. In the first study, injection of urinary SC-EVs into STZ-induced rats improved renal function decline, glomerular structure, decreased glomerular damage markers, and reduced the kidney expression of TGF- $\beta 1$ [21]. Secretion of miR-16-5p from uSC-EVs ameliorated glucose-induced podocyte injury by targeting VEGF signalling, whereas administration of miR-16-5p-uSCs led to a decrease in the levels of kidney VEGFA, TGF- $\beta 1$, and pro-inflammatory mediators (MCP-1, TNF- $\alpha$ ), which were increased following the establishment of DN. In the second study by this group, the addition of MSC-EVs containing miR-26a-5p into human podocytes inhibited TLR4 and NF-кB/VEGFA signalling induced by glucose [20]. In line with this, the injection of miR-26a-5pEVs decreased glomerular structural abnormalities in $\mathrm{db} /$ $\mathrm{db}$ mice. In another study in $\mathrm{db} / \mathrm{db}$ mice, MSC-EVs ameliorated renal functional decline, improved podocyte damage, and inhibited apoptosis. [30]. These effect were mediated through miR-486 and inducing autophagy flux through modulation of the Smad1/mTOR signalling pathway. Further, SC-EVs express miRNAs involved in vasculature protection and secrete growth factors, which decrease endothelial cell (EC) damage. Chen et al. showed that the delivery of GNDF from MSC-EVs activated the kidney SIRT1/eNOS pathway, resulting in significantly decreased IF in UUO mice [11]. Those GNDF-MSC-EVs decreased apoptosis, and induced angiogenic activities in vitro. Further, EPSs were documented to reduce glomerular mesangial, and endothelial cell injury and to enhance microvascular repair in AKI and CKD [18]. Finally, AFSC-EVs were shown to mediate antiapoptotic, and pro-angiogenic effects in mice with Alport Syndrome [36].

The anti-oxidant response was also involved in affording protection following EVs administration in CKD [37, 44, 47]. However, the involved mediators were not investigated, except for one study by Zou et al. [47]. This study demonstrated that kidney ST-like cell-EVs transfer mitochondria, which remain functional in the recipient TECs. Administration of these EVs improved oxidative stress, decreased cell damage, and improved kidney perfusion.

The graphical representation of the current concept of EV-enclosed active molecules based on their downstream mechanism in the kidney is shown in Fig. 4, and the detailed characteristic of study design of the experiments evaluating particular EV effector molecules is summarised in Table 2. Also, the results of our in silico analysis showing (A) the overlap in the expression patterns of the protective miRNAs in EVs from different cell sources (mesenchymal stem cells, endothelial cells, and urine, and (B) target-target interaction network among miRNA targets with underling KEGG ontologies and GO terms, are shown on Fig. 5.

\section{Discussion}

Our qualitative review shows that the administration of EVs has consistently ameliorated functional, structural, and molecular measurements in studies regarding progressive kidney disease. The published data imply that SC-EVs mediate nephroprotection by influencing kidney fibrotic genes and exerting immunomodulatory and cell-protective activities. Additionally, our meta-analysis confirmed an improvement in renal function decline in CKD animals receiving EV-based treatment in comparison to untreated CKD controls. A major advantage of using EVs over stem cells themselves is to avoid the potential risks of tumorogenesis or maldifferentiation of the engrafted cells. EVs were postulated to represent a less immunogenic, and non-mutagenic to the recipient compared with other gene delivery vehicles [50]. Thus, we suggest EV-based regenerative approaches could offer a safer 'off-shelf' therapy for patients with CKD.

Knowing the particular type of study design and the factors influencing the efficacy of EV-based treatment may facilitate future experimental studies and may help design studies in specific patient populations. Thus, for the studies with available creatinine and urea data, we performed univariable stratified meta-analyses to investigate candidate predictors for EV-based therapy effectiveness, by CKD setting. In our findings, the differences in the functional efficacy of EV therapy appear to be model dependent. A considerable proportion of the animal records included in our analysis involved diabetic CKD models. Despite the current recommended multidisciplinary treatment including intensive glycemic control, tight blood pressure control, and renoprotective therapy such as renin-angiotensin-aldosteron inhibitors and sodium-glucose transport protein 2 inhibitors [51], there is still large residual risk of CKD progression in people with diabetes [52], indicating that additional CKD prevention options for people with diabetes are needed. The results of our meta-analysis show EV therapy is a promising approach for CKD progression in experimental diabetes and may be worthy to consider the possibility of clinical application. The calculated treatment effects were considerably weaker in other CKD aetiologies, which is in line with a results of a previous analysis by Papazova et al., who evaluated stem cell therapies in various pre-clinical models of CKD [16]. However, their study did not included treatment with EVs.

Secondly, we observe significant differences in the functional efficacy of EVs between the preventive and curative character of EV administration. Among the studies included, several (studies in UUO and two studies in type 1 diabetes), have utilized preventive EV administration. Their findings varied regarding the effect on renal decline outcomes, with a considerable proportion failing to document that administered EVs have significantly reduced plasma urea or plasma 
creatinine concentrations [38, 10, 29]. Moreover, the recent study performed in a rat model of toxin-induced CKD [12] has reported an increased effect of MSCs and MSC-EVs on kidney function decline/renal histology to be associated with later treatment administration, associating kidney inflammation as the main factor to renoprotective effects of these treatments. This is in agreement with the results from experimental studies that documented that the stem cell-EV uptake in the normal kidney tissue is very limited and could be specifically induced with a more severe tissue injury [19, $23,28,43,53]$. Several in vivo (or ex vivo) studies have been performed regarding EV biodistribution in AKI [53] and CKD-UUO animals [19, 28, 43], to identify that intravenously injected EVs were specifically retained by the damaged kidney and were engrafted only to a limited (20 times less) extent by the non-affected kidney [28]. Those EVs homed to TECs or PTCs, while fewer EVs were detected in the glomeruli (reviewed in 54). Moreover, animal studies in HT have shown that, following intra-renal administration, EVs engrafted specifically into the post-stenotic kidney, and that these EVs accumulated in TECs, and in kidney infiltrating macrophages [23]. Concurrently, the specificity of EVs towards homing in injured kidney was hypothesized to involve infiltrating leucocytes or increased surface markers on parenchymal kidney cells during inflammation [54]. However, the existence of complex types of communication between EVs and kidney cells including various receptors/ ligands implicated has been suggested. As one mechanism to enter cells, EV express MSC surface adhesion molecules, such as tetraspanins (CD44, CD29). It has been shown that treatment with antibodies against those $\mathrm{CD}$ molecules prevented MSC-EVs from entering TECs [55, 56]. Conversely, recent data suggested that EVs rely on phosphatidylserine as target receptors, and characterized the T cell Ig and mucin domain-containing family molecules (TIMs) as receptors binding EVs [57]. On Tcells, EVs can directly interact with the T-cell membrane receptor Tim-4 $[57,59]$. In the kidney, another TIM family molecule - Tim-1 (or kidney injury molecule -1) is specifically expressed in the damaged epithelium [58]. KIM-1 expression is undetectable in the normal kidney, while it specifically increases in TEC membranes during the early stage of AKI, conferring them phagocytic functions [60] while in progressive CKD, KIM-1 increases gradually with the disease stage, and it is involved in mediating the inflammatory response. This supports the notion that availability of kidney TIM-1 (and leucocyte TIM-4) may be another mediator of EV therapeutic properties [61, 62]. Moreover, conjugation of EVs with the KIM-1 targeting antibody has been shown to enhance the retention of exogenously administered EVs in the kidney and endowed with increased therapeutic anti-inflammatory properties in murine renal artery stenosis [63]. Nevertheless, the complete mechanisms behind the interaction between EVs and injured kidney cells remain poorly understood, are complex, and involve many mediating factors. Together, the gathered data imply the need for a better understanding of EV uptake, and the need for new approaches to enable specifically targeting retention, and uptake of EVs into the kidney cells [64]. We believe, though that future genetic and structural modifications of the isolated EVs will accelerate therapeutic application and will further improve the EV-based therapy efficacy.

Third, multiple EV-doses were considerably more effective in reducing renal decline. Accordingly, it would be worthy to model the relationship between the total EV dosage and the magnitude of the therapeutic effect. This task, however; requires complete, standardized dataset with regards to the dosing (f.ex the number of particles used (per body mass)), and as such we postulate this issue should be resolved by further studies. Phase 1 trials may be designed to address the safety of EV treatment, specifically focusing on EV pharmacokinetic profile, and establish the amount of EVs that causes the therapeutic effect without inducing toxicity.

Experimental studies have not only implicated stem cellderived EVs in kidney protection but also pointed to the role of EV-enclosed miRNAs [65]. Since the miRNA depleted EVs do not present any protective properties in the kidney injury, it is likely that those miRNAs contribute to the kidney cell reprogramming [65]. Based on these findings, stem cell-derived miRNAs were identified as effectors of the EV nephroprotective benefits, implicated in inhibiting fibrosis and cell damage pathways in CKD setting. Nethertheless, many of the studies focused on isolated microRNA-gene (or gene-gene) relations and did not address the complexity of their interactions. As the protective effect of EVs may be a result of multiple miRNAs, a more comprehensive understanding of the global mechanisms and related EV secretome is needed. Additionally, the exact pathways involved in the immune-modulatory effects EVs in CKD may also need further investigation. In the stratified analyses, the therapeutic effect of EV-based treatment on renal decline was considerably stronger in diabetic than in non-diabetic animals. This differences in $\mathrm{EV}$ effectiveness might be due the underlying differences in study design, or may be attributed to antidiabetic properties of EVs. Noteworthy, the treatment with MSC-EVs did not significantly affect insulin tolerance [15, $23,24]$ or ameliorate hyperglycemia [21, 25, 29], indicating that inflammation or other mechanism altered by EVs may play role, at least in this particular setting.

The results of our analyses prompted us to highlight the relevant areas for future research. Several features, such as the CKD model, time of EV administration, and EV dose appeared to have considerable effects on therapy effectiveness in our univariable analyses. However, definitive conclusions about independent predictors of EVs efficacy cannot be made at this moment due to high variance and a relatively 
small number of the available studies. This also implies that the EV-based therapy is not yet ready for clinical application and that further data are needed to design the optimal intervention, create comprehensive experimental protocols, and to incorporate these protocols into clinical setting. Our findings are less clear in several other areas. First, it was not possible to investigate whether a certain EV type or combinations of these are most effective. EVs represent a heterogeneous collection and it remains to be determined which subpopulations confer the protective effects, their target pathways, and the specific molecules they interact with their targets. Also, when designing future studies, several methodological aspects should be taken into consideration: (1) improvement of the study quality (f.ex., by using randomisation into study groups) (2) continuous, growing adherence to the ISEV guidelines, which is needed for standardisation and comparison between studies [66]; (3) use of a gradient of EV doses in animal models; (4) extension of the assessment of kidney function to extrapolate the treatment effect into later CKD stages; and (5) evaluation of EV molecular content at rigorous purity of $\mathrm{EV}$ preparations (it was documented that miRNA to EV ratios are low and that in cell culture supernatants or biofluids, a large fraction of miRNAs is present outside EVs [67-70]). Finally, limited data are available on the long-term structural outcomes and safety of EV administration, and about the molecular effects of early versus later dosing of EVs in experimental CKD.

Several limitations of this study should be mentioned. The results of every meta-analysis are determined by the quality and quantity of the original studies. Our findings are based on a number of heterogeneous studies, interventions, and outcomes, which is inherent in animal research. Most experimental studies in CKD used protocols that differ from model to model and between laboratories, in addition to the heterogeneity inherent in different EV subtypes. Furthermore, a significant proportion of the studies did not report according to ISEV standards. The relatively small number of studies and large heterogeneity also indicates that the conclusions about the effectiveness of EV-therapeutic strategies should be interpreted with caution. Finally, the studies included in this review focused on the protective properties of populations of EVs, that were mainly derived from MSCs and it is not known how these results relate to other EV populations. For example, endothelial cell-EVs were recently shown to attenuate cardiac ischemic injury [71] and they express a protective miRNA signature. All these issues represent areas for future research.

Despite these limitations, our manuscript is the first report that systematically aggregates the data regarding the functional nephroprotective properties of $\mathrm{EV}$ s in pre-clinical studies of CKD, connects these data with molecular findings, and summarises the current state of the EV-biomarker field in experimental CKD. Collectively, the included literature demonstrates that the concept of EV-based treatment for $\mathrm{CKD}$, remains a promising one, but that more research is needed regarding standardisation of $\mathrm{EV}$ protocols, improving study quality, determining the optimal EV delivery and dosage, and most importantly, understanding the global biological mechanisms of the observed protective effects.

Supplementary Information The online version contains supplementary material available at https://doi.org/10.1007/s12015-021-10189-9.

Authors' Contributions All authors contributed to the study design, partake in the literature search, and approved the final version of the manuscript. N.N. collected the data, performed data analysis, interpreted the results, and wrote the manuscript. M.Y. and E.S. interpreted the results, contributed to writing and editing of the manuscript, figures and tables.

Funding $\mathrm{NN}$ is supported by the university statutory fund (no. SUB/1/ DN/20/002/4407).

Data Availability The dataset analysed in the manuscript can be obtained from author on reasonable request.

\section{Declarations}

Ethics Approval The manuscript is a systematic review and do not involve humans or animals.

Conflicts of Interest/Competing Interests The authors have no relevant financial or non-financial interests to disclose.

Open Access This article is licensed under a Creative Commons Attribution 4.0 International License, which permits use, sharing, adaptation, distribution and reproduction in any medium or format, as long as you give appropriate credit to the original author(s) and the source, provide a link to the Creative Commons licence, and indicate if changes were made. The images or other third party material in this article are included in the article's Creative Commons licence, unless indicated otherwise in a credit line to the material. If material is not included in the article's Creative Commons licence and your intended use is not permitted by statutory regulation or exceeds the permitted use, you will need to obtain permission directly from the copyright holder. To view a copy of this licence, visit http://creativecommons.org/licenses/by/4.0/.

\section{References}

1. GBD Chronic Kidney Disease Collaboration. (2020). Global, regional, and national burden of chronic kidney disease, 19902017: a systematic analysis for the Global Burden of Disease Study 2017 [published online February 13, 2020]. Lancet. Cockwell $\mathrm{P}$ and Fisher L. The global burden of chronic kidney disease [published online February 13, 2020]. https://doi.org/10.1016/ S0140-6736(19)32977-0

2. Ruiz-Ortega, M., Rayego-Mateos, S., Lamas, S., Ortiz, A., \& Rodrigues-Diez, R. R. (2020). Targeting the progression of chronic kidney disease. Nature Reviews Nephrology, 16(5), 269-288.

3. Breyer, M. D., \& Susztak, K. (2016). Developing Treatments for Chronic Kidney Disease in the 21st Century. Seminars in Nephrology, 36(6), 436-447. 
4. Ix, J. H., \& Shlipak, M. G. (2018). Trial of pirfenidone to prevent progression of chronic kidney disease. (available online at http:// grantome.com/grant/NIH/U01-DK111510-01A1)

5. van der Pol, E., Böing, A. N., Gool, E. L., \& Nieuwland, R. (2016). Recent developments in the nomenclature, presence, isolatin, detection and clinical impact of extracellular vesicles. Journal of Thrombosis and Haemostasis, 14(1), 48-56.

6. Ratajczak, J., Miekus, K., Kucia, M., Zhang, J., Reca, R., Dvorak, P., \& Ratajczak, M. Z. (2006). Embryonic stem cell-derived microvesicles reprogram hematopoietic progenitors: evidence for horizontal transfer of mRNA and protein delivery. Leukemia, 20(5), 847-856.

7. Fais, S., O’Driscoll, L., Borras, F. E., Buzas, E., Camussi, G., Cappello, F., et al. (2016). Evidence-Based Clinical Use of Nanoscale Extracellular Vesicles in Nanomedicine. ACS Nano, 10(4), 3886-3899.

8. Tomasoni, S., Longaretti, L., Rota, C., Morigi, M., Conti, S., Gotti, E., et al. (2013). Transfer of growth factor receptor mRNA via exosomes unravels the regenerative effect of mesenchymal stem cells. Stem Cells and Development, 22(5), 772-780.

9. Bruno, S., Porta, S., \& Bussolati, B. (2016). Extracellular vesicles in renal tissue damage and regeneration. European Journal of Pharmacology, 790, 83-91.

10. Chen, L., Wang, Y., Li, S., Zuo, B., Zhang, X., Wang, F., \& Sun, D. (2020). Exosomes derived from GDNF-modified human adipose mesenchymal stem cells ameliorate peritubular capillary loss in tubulointerstitial fibrosis by activating the SIRT1/eNOS signaling pathway. Theranostics, 10(20), 9425-9442.

11. van Koppen, A., Joles, J. A., van Balkom, B. W., Lim, S. K., de Kleijn, D., Giles, R. H., \& Verhaar, M. C. (2012). Human embryonic mesenchymal stem cell-derived conditioned medium rescues kidney function in rats with established chronic kidney disease. PLoS One, 7(6), e38746.

12. Ramírez-Bajo, M. J., Martín-Ramírez, J., Bruno, S., Pasquino, C., Banon-Maneus, E., Rovira, J., et al. (2020). Nephroprotective Potential of Mesenchymal Stromal Cells and Their Extracellular Vesicles in a Murine Model of Chronic Cyclosporine Nephrotoxicity. Frontiers in Cell and Developmental Biology, 8, 296.

13. Collino, F., Pomatto, M., Bruno, S., Lindoso, R. S., Tapparo, M., Sicheng, W., et al. (2017). Exosome and Microvesicle-Enriched Fractions Isolated from Mesenchymal Stem Cells by Gradient Separation Showed Different Molecular Signatures and Functions on Renal Tubular Epithelial Cells. Stem Cell Reviews and Reports, 13(2), 226-243.

14. Song, T., Eirin, A., Zhu, X., Zhao, Y., Krier, J. D., Tang, H., et al. (2020). Mesenchymal Stem Cell-Derived Extracellular Vesicles Induce Regulatory T Cells to Ameliorate Chronic Kidney Injury. Hypertension, 75(5), 1223-1232.

15. Moher, D., Liberati, A., Tetzlaff, J., Altman, D. G., \& PRISMA Group. (2009). Preferred reporting items for systematic reviews and meta-analyses: the PRISMA statement. PLoS Medicine, 6(7), e1000097.

16. Papazova, D. A., Oosterhuis, N. R., Gremmels, H., van Koppen, A., Joles, J. A., \& Verhaar, M. C. (2015). Cell-based therapies for experimental chronic kidney disease: a systematic review and meta-analysis. Disease Models \& Mechanisms, 8(3), 281-293.

17. Cambier, L., Giani, J. F., Liu, W., Ijichi, T., Echavez, A. K., Valle, J., \& Marbán, E. (2018). Angiotensin II-Induced End-Organ Damage in Mice Is Attenuated by Human Exosomes and by an Exosomal Y RNA Fragment. Hypertension, 72(2), 370-380.

18. Cantaluppi, V., Medica, D., Mannari, C., Stiaccini, G., Figliolini, F., Dellepiane, S., et al. (2015). Endothelial progenitor cellderived extracellular vesicles protect from complement-mediated mesangial injury in experimental anti-Thy 1.1 glomerulonephritis. Nephrology, Dialysis, Transplantation : Official Publication of the
European Dialysis and Transplant Association - European Renal Association, 30(3), 410-422.

19. Choi, H. Y., Lee, H. G., Kim, B. S., Ahn, S. H., Jung, A., Lee, M., et al. (2015). Mesenchymal stem cell-derived microparticles ameliorate peritubular capillary rarefaction via inhibition of endothelial-mesenchymal transition and decrease tubulointerstitial fibrosis in unilateral ureteral obstruction. Stem Cell Research \& Therapy, 6(1), 18.

20. Duan, Y. R., Chen, B. P., Chen, F., Yang, S. X., Zhu, C. Y., Ma, Y. L., et al. (2019). Exosomal microRNA-16-5p from human urinederived stem cells ameliorates diabetic nephropathy through protection of podocyte. Journal of Cellular and Molecular Medicine (Advance online publication available at https://doi.org/10.1111/ jcmm.14558)

21. Duan, Y., Luo, Q., Wang, Y., Ma, Y., Chen, F., Zhu, X., et al. (2020). Adipose mesenchymal stem cell-derived extracellular vesicles containing microRNA-26a-5p target TLR4 and protect against diabetic nephropathy. The Journal of Biological Chemistry, 295(37), 12868-12884.

22. Ebrahim, N., Ahmed, I. A., Hussien, N. I., Dessouky, A. A., Farid, A. S., Elshazly, A. M., et al. (2018). Mesenchymal Stem Cell-Derived Exosomes Ameliorated Diabetic Nephropathy by Autophagy Induction through the mTOR Signaling Pathway. Cells, 7(12), 226.

23. Eirin, A., Zhu, X. Y., Puranik, A. S., Tang, H., McGurren, K. A., van Wijnen, A. J., et al. (2017). Mesenchymal stem cell-derived extracellular vesicles attenuate kidney inflammation. Kidney International, 92(1), 114-124.

24. Eirin, A., Ferguson, C. M., Zhu, X. Y., Saadiq, I. M., Tang, H., Lerman, A., \& Lerman, L. O. (2020). Extracellular vesicles released by adipose tissue-derived mesenchymal stromal/stem cells from obese pigs fail to repair the injured kidney. Stem Cell Research, 47, 101877.

25. Grange, C., Tritta, S., Tapparo, M., Cedrino, M., Tetta, C., Camussi, G., \& Brizzi, M. F. (2019). Stem cell-derived extracellular vesicles inhibit and revert fibrosis progression in a mouse model of diabetic nephropathy. Scientific Reports, 9(1), 4468.

26. He, J., Wang, Y., Sun, S., Yu, M., Wang, C., Pei, et al. (2012). Bone marrow stem cells-derived mi-crovesicles protect against renal injury in the mouse remnant kidney model. Nephrology (Carlton, Vic.), 17(5), 493-500.

27. He, J., Wang, Y., Lu, X., Zhu, B., Pei, X., Wu, J., \& Zhao, W. (2015). Micro-vesicles derived from bone marrow stem cells protect the kidney both in vivo and in vitro by microRNA-dependent repairing. Nephrology (Carlton, Vic.), 20(9), 591-600.

28. Ji, C., Zhang, J., Zhu, Y., Shi, H., Yin, S., Sun, F., et al. (2020). Exosomes derived from hucMSC attenuate renal fibrosis through CK18/ $\beta$-TRCP-mediated YAP degradation. Cell Death \& Disease, $11,327$.

29. Jiang, Z. Z., Liu, Y. M., Niu, X., Yin, J. Y., Hu, B., Guo, S. C., et al. (2016). Exosomes secreted by human urine-derived stem cells could prevent kidney complications from type I diabetes in rats. Stem Cell Research \& Therapy, 7, 24.

30. Jin, J., Shi, Y., Gong, J., Zhao, L., Li, Y., He, Q., \& Huang, H. (2019). Exosome secreted from adiposederived stem cells attenuates diabetic nephropathy by promoting autophagy flux and inhibiting apoptosis in podocyte. Stem Cell Research \& Therapy, 10(1), 95.

31. Jin, J., Wang, Y., Zhao, L., Zou, W., Tan, M., \& He, Q. (2020). Exosomal miRNA-215-5p Derived from Adipose-Derived Stem Cells Attenuates Epithelial-Mesenchymal Transition of Podocytes by Inhibiting ZEB2. BioMed Research International, 2020, 2685305.

32. Kholia, S., Herrera Sanchez, M. B., Cedrino, M., Papadimitriou, E., Tapparo, M., Deregibus, M. C., et al. (2018). Human Liver 
Stem Cell-Derived Extracellular Vesicles Prevent Aristolochic Acid-Induced Kidney Fibrosis. Frontiers in Immunology, 9, 1639.

33. Kholia, S., Herrera Sanchez, M. B., Cedrino, M., Papadimitriou, E., Tapparo, M., Deregibus, M. C., et al. (2020). Mesenchymal Stem Cell Derived Extracellular Vesicles Ameliorate Kidney Injury in Aristolochic Acid Nephropathy. Frontiers in Cell and Developmental Biology, 8, 188.

34. Lindoso, R. S., Lopes, J. A., Binato, R., Abdelhay, E., Takiya, C. M., Miranda, K. R., et al. (2019). Adipose Mesenchymal CellsDerived EVs Alleviate DOCA-Salt-Induced Hypertension by Promoting Cardio-Renal Protection. Molecular Therapy. Methods \& Clinical Development, 16, 63-77.

35. Nagaishi, K., Mizue, Y., Chikenji, T., Otani, M., Nakano, M., Konari, N., \& Fujimiya, M. (2016). Mesenchymal stem cell therapy ameliorates diabetic nephropathy via the paracrine effect of renal trophic factors including exosomes. Scientific Reports, 6 , 34842.

36. Sedrakyan, S., Villani, V., Da Sacco, S., Tripuraneni, N., \& Achena, A. (2017). Amniotic fluid stem cell-derived vesicles protect from VEGF-induced endothelial damage. Scientific Reports, 7,16875 .

37. Shi, Z., Wang, Q., Zhang, Y., \& Jiang, D. (2020). Extracellular vesicles produced by bone marrow mesenchymal stem cells attenuate renal fibrosis, in part by inhibiting the RhoA/ROCK pathway, in a UUO rat model. Stem Cell Research \& Therapy, 11(1), 253.

38. Wang, Y., Guo, Y. F., Fu, G. P., Guan, C., Zhang, X., Yang, D. G., \& Shi, Y. C. (2020). Protective effect of miRNA-containing extracellular vesicles derived from mesenchymal stromal cells of old rats on renal function in chronic kidney disease. Stem Cell Research \& Therapy, 11(1), 274.

39. Wang, Y., Lu, X., He, J., \& Zhao, W. (2015). Influence of erythropoietin on microvesicles derived from mesenchymal stem cells protecting renal function of chronic kidney disease. Stem Cell Research \& Therapy, 6(1), 100.

40. Wang, B., Yao, K., Huuskes, B. M., Shen, H. H., Zhuang, J., Godson, C., et al. (2016). Mesenchymal Stem Cells Deliver Exogenous MicroRNA-let7c via Exosomes to Attenuate Renal Fibrosis. Molecular Therapy : The Journal of the American Society of Gene Therapy, 24(7), 1290-1301.

41. Wang, H., Wang, B., Zhang, A., Hassounah, F., Seow, Y., Wood, M., et al. (2019). Exosome-Mediated miR-29 Transfer Reduces Muscle Atrophy and Kidney Fibrosis in Mice. Molecular Therapy: The Journal of the American Society of Gene Therapy, 27(3), 571-583.

42. Yang, J., Wang, M., Zhu, F., Sun, J., Xu, H., Shin, Chong Lee, O. L., et al. (2019). Putative endothelial progenitor cells do not promote vascular repair but attenuate pericyte-myofibroblast transition in UUO-induced renal fibrosis. Stem Cell Research \& Therapy, $10(1), 104$.

43. Zhang, A., Wang, H., Wang, B., Yuan, Y., Klein, J. D., \& Wang, X. H. (2019). Exogenous miR-26a suppresses muscle wasting and renal fibrosis in obstructive kidney disease. FASEB Journal : Official Publication of the Federation of American Societies for Experimental Biology, 33(12), 13590-13601.

44. Zhang, G., Yu, S., Sun, S., Zhang, L., Zhang, G., \& Xu, L. (2019). Extracellular-vesicles derived from human Wharton-Jelly mesenchymal stromal cells ameliorated cyclosporin A-induced renal fibrosis in rats. The International Journal of Biochemistry \& Cell Biology, 12(7), 8943-8949.

45. Zhao, Y., Zhu, X., Zhang, L., Ferguson, C. M., Song, T., Jiang, K., et al. (2020). Mesenchymal Stem/Stromal Cells and their Extracellular Vesicle Progeny Decrease Injury in Poststenotic Swine Kidney Through Different Mechanisms. Stem Cells and Development, 29(18), 1190-1200.
46. Zhong, L., Liao, G., Wang, X., Li, L., Zhang, J., Chen, Y., et al. (2019). Mesenchymal stem cells-microvesicle-miR-451a ameliorate early diabetic kidney injury by negative regulation of P15 and P19. Experimental Biology and Medicine (Maywood, N.J.), First published January 6, 2019 (Advance online publication available at: https://doi.org/10.1177/1535370218819726)

47. Zou, X., Kwon, S. H., Jiang, K., Ferguson, C. M., Puranik, A. S., Zhu, X., \& Lerman, L. O. (2018). Renal scattered tubular-like cells confer protective effects in the stenotic murine kidney mediated by release of extracellular vesicles. Scientific Reports, 8(1), 1263.

48. Théry, C., Witwer, K. W., Aikawa, E., Alcaraz, M. J., Anderson, J. D., Andriantsitohaina, R., et al. (2018). Minimal information for studies of extracellular vesicles 2018 (MISEV2018): a position statement of the International Society for Extracellular Vesicles and update of the MISEV2014 guidelines. Journal of Extracellular Vesicles, 7(1), 1535750.

49. Macleod, M. R., O'Collins, T., Howells, D. W., et al. (2004). Pooling of animal experimental data reveals influence of study design and publication bias. Stroke, 35, 1203-8.

50. Wiklander, O., Brennan, M. Á., Lötvall, J., Breakefield, X. O., \& El Andaloussi, S. (2019). Advances in therapeutic applications of extracellular vesicles. Science Translational Medicine, 11(492), eaav8521.

51. Buse, J. B., Wexler, D. J., Tsapas, A., Rossing, P., Mingrone, G., \& Mathieu, C. (2020). 2019 Update to: Management of Hyperglycemia in Type 2 Diabetes, 2018. A Consensus Report by the American Diabetes Association (ADA) and the European Association for the Study of Diabetes (EASD). Diabetes Care, 43(2), 487-493.

52. Gaede, P., Vedel, P., Larsen, N., Jensen, G. V., Parving, H. H., \& Pedersen, O. (2003). Multifactorial intervention and cardiovascular disease in patients with type 2 diabetes. The New England Journal of Medicine, 348(5), 383-393.

53. Choi, H. Y., Moon, S. J., Ratliff, B. B., Ahn, S. H., Jung, A., \& Park, H. C. (2014). Microparticles from kidney-derived mesenchymal stem cells act as carriers of proangiogenic signals and contribute to recovery from acute kidney injury. PloS One, 9(2), e87853.

54. Aghajani Nargesi, A., Lerman, L. O., \& Eirin, A. (2017). Mesenchymal stem cell-derived extracellular vesicles for kidney repair: current status and looming challenges. Stem Cell Research \& Therapy, 8(1), 273.

55. Lindoso, R. S., Collino, F., Bruno, S., Araujo, D. S., \& Sant'Anna, \& Camussi, G. . (2014). Extracellular vesicles released from mesenchymal stromal cells modulate miRNA in renal tubular cells and inhibit ATP depletion injury. Stem Cells and Development, 23(15), 1809-1819.

56. Grange, C., Tapparo, M., Bruno, S., et al. (2014). Biodistribution of mesenchymal stem cell-derived extracellular vesicles in a model of acute kidney injury monitored by optical imaging. International Journal of Molecular Medicine, 33(5), 1055-1063.

57. Miyanishi, M., Tada, K., Koike, M., Uchiyama, Y., Kitamura, T., \& Nagata, S. (2007). Identification of Tim4 as a phosphatidylserine receptor. Nature, 450(7168), 435-9. https://doi.org/10.1038/ nature 06307

58. Ichimura, T., Asseldonk, E. J., Humphreys, B. D., Gunaratnam, L., Duffield, J. S., \& Bonventre, J. V. (2008). Kidney injury molecule-1 is a phosphatidylserine receptor that confers a phagocytic phenotype on epithelial cells. The Journal of Clinical Investigation, 118(5), 1657-68.

59. van Dongen HM, Masoumi N, Witwer KW, Pegtel DM. Extracellular Vesicles Exploit Viral Entry Routes for Cargo Delivery. Microbiology and Molecular Biology Reviews, 280, 369-86. https://doi.org/10.1128/MMBR.00063-15 
60. Yang, L., Brooks, C. R., Xiao, S., Sabbisetti, V., Yeung, M. Y., Hsiao, L. L., Ichimura, T., Kuchroo, V., \& Bonventre, J. V. (2015). KIM-1-mediated phagocytosis reduces acute injury to the kidney. The Journal of Clinical Investigation, 125(4), 1620-36. https://doi.org/10.1172/JCI75417

61. Sabbisetti, V. S., Waikar, S. S., Antoine, D. J., Smiles, A., Wang, C., Ravisankar, A., Ito, K., Sharma, S., Ramadesikan, S., Lee, M., Briskin, R., De Jager, P. L., Ngo, T. T., Radlinski, M., Dear, J. W., Park, K. B., Betensky, R., Krolewski, A. S., \& Bonventre, J. V. (2014). Blood kidney injury molecule-1 is a biomarker of acute and chronic kidney injury and predicts progression to ESRD in type I diabetes. Journal of the American Society of Nephrology, 25(10), 2177-86.

62. Bonventre, J. V. (2012). Can we target tubular damage to prevent renal function decline in diabetes? Seminars in Nephrology, 32(5), $452-62$.

63. Chen, X. J., Jiang, K., Ferguson, C. M., Tang, H., Zhu, X., Lerman, A., \& Lerman, L. O. (2020). Augmented efficacy of exogenous extracellular vesicles targeted to injured kidneys. Signal Transduction and Targeted Therapy, 14(5), 199. https://doi.org/ 10.1038/s41392-020-00304-6

64. Choi, H., \& Lee, D. S. (2016). Illuminating the physiology of extracellular vesicles. Stem Cell Research \& Therapy, 7(1), 55.

65. Collino, F., Bruno, S., Incarnato, D., Dettori, D., Neri, F., Provero, P., et al. (2015). AKI Recovery Induced by Mesenchymal Stromal Cell-Derived Extracellular Vesicles Carrying MicroRNAs. Journal of the American Society of Nephrology: JASN, 26(10), 2349-2360.

66. Mateescu, B., Kowal, E. J., van Balkom, B. W., Bartel, S., Bhattacharyya, S. N., Buzás, E. I., et al. (2017). Obstacles and opportunities in the functional analysis of extracellular vesicle RNA - an ISEV position paper. Journal of Extracellular Vesicles, 6(1), 1286095.

67. Turchinovich, A., Tonevitsky, A. G., Cho, W. C., \& Burwinkel, B. (2015). Check and mate to exosomal extracellular miRNA: new lesson from a new approach. Frontiers in Molecular Biosciences, 2,11 .

68. Arroyo, J. D., Chevillet, J. R., Kroh, E. M., Ruf, I. K., Pritchard, C. C., Gibson, D. F., et al. (2011). Argonaute2 complexes carry a population of circulating microRNAs independent of vesicles in human plasma. Proceedings of the National Academy of Sciences of the United States of America, 108(12), 5003-5008.

69. Chevillet, J. R., Kang, Q., Ruf, I. K., Briggs, H. A., Vojtech, L. N., Hughes, S. M., et al. (2014). Quantitative and stoichiometric analysis of the microRNA content of exosomes. Proceedings of the National Academy of Sciences of the United States of America, 111(41), 14888-14893.

70. Albanese, M., Chen, A., Hüls, C., Gärtner, K., Tagawa, T., Keppler O.T., et al. (2020). Micro RNAs are minor constituents of extracellular vesicles and are hardly delivered to target cells. (Available online at https://doi.org/10.1101/2020.05.20.106393)

71. Yadid, M., Lind, J. U., Ardoña, H., Sheehy, S. P., Dickinson, L. E., Eweje, F., et al. (2020). Endothelial extracellular vesicles contain protective proteins and rescue ischemia-reperfusion injury in a human heart-on-chip. Science Translational Medicine, 12(565), eaax 8005 .

Publisher's Note Springer Nature remains neutral with regard to jurisdictional claims in published maps and institutional affiliations. 